\title{
On consistent micromechanical estimation of macroscopic elastic energy, coherence energy and phase transformation strains for SMA materials
}

Received: 15 February 2016 / Accepted: 7 September 2016 / Published online: 22 September 2016

(C) The Author(s) 2016. This article is published with open access at Springerlink.com

\begin{abstract}
An apparatus of micromechanics is used to isolate the key ingredients entering macroscopic Gibbs free energy function of a shape memory alloy (SMA) material. A new self-equilibrated eigenstrains influence moduli (SEIM) method is developed for consistent estimation of effective (macroscopic) thermostatic properties of solid materials, which in microscale can be regarded as amalgams of $n$-phase linear thermoelastic component materials with eigenstrains. The SEIM satisfy the self-consistency conditions, following from elastic reciprocity (Betti) theorem. The method allowed expressing macroscopic coherency energy and elastic complementary energy terms present in the general form of macroscopic Gibbs free energy of SMA materials in the form of semilinear and semiquadratic functions of the phase composition. Consistent SEIM estimates of elastic complementary energy, coherency energy and phase transformation strains corresponding to classical Reuss and Voigt conjectures are explicitly specified. The Voigt explicit relations served as inspiration for working out an original engineering practice-oriented semiexperimental SEIM estimates. They are especially conveniently applicable for an isotropic aggregate (composite) composed of a mixture of $n$ isotropic phases. Using experimental data for NiTi alloy and adopting conjecture that it can be treated as an isotropic aggregate of two isotropic phases, it is shown that the NiTi coherency energy and macroscopic phase strain are practically not influenced by the difference in values of austenite and martensite elastic constants. It is shown that existence of nonzero fluctuating part of phase microeigenstrains field is responsible for building up of so-called stored energy of coherency, which is accumulated in pure martensitic phase after full completion of phase transition. Experimental data for NiTi alloy show that the stored coherency energy cannot be neglected as it considerably influences the characteristic phase transition temperatures of SMA material.
\end{abstract}

Keywords SMA - NiTi alloys - Adaptive composite - Macroscopic free energy functions - Gibbs energy · Micromechanics - Coherence energy - Stored coherency energy - Ultimate phase transformation eigenstrains . Self-equilibrated eigenstrains influence moduli - SEIM - Effective property estimates - Martensitic phase transformation

$$
\begin{aligned}
& \text { Notation } \\
& \quad \begin{array}{l}
\boldsymbol{1} \leftrightarrow \delta_{i j}, \quad(\boldsymbol{I})_{k l m n} \leftrightarrow 0.5\left(\delta_{k m} \delta_{l n}+\delta_{k n} \delta_{l m}\right), \quad(! \alpha) \rightarrow \text { No summation over index } \alpha \\
\boldsymbol{A}^{\mathrm{T}}\left(A_{k l}^{\mathrm{T}}=A_{l k} \text { or } A_{k l m n}^{\mathrm{T}}=A_{m n k l}\right), \quad \boldsymbol{A}^{-1}\left(A_{k l}^{-1} A_{l m}=\delta_{k m} \text { or } A_{k l m n}^{-1} A_{m n p r}=I_{k l p r}\right), \\
\boldsymbol{A B}\left(A_{k l} B_{l m} \text { or } A_{k l m n} B_{m n p r}\right), \quad \mathbf{A} \cdot \mathbf{B}=\operatorname{tr}\left(\mathbf{A B}^{\mathrm{T}}\right) \quad\left(A_{i j} B_{i j} \text { or } A_{k l m n} B_{k l m n}\right)
\end{array}
\end{aligned}
$$

The coming into existence of the present paper would not be possible without contributions and long discussions with Professor Bogdan Raniecki who passed away suddenly on August 2014. I dedicate this work to his memory.

Communicated by Andreas Öchsner.

A. Ziółkowski $(\varangle)$

Institute of Fundamental Technological Research, Polish Academy of Sciences, Pawinskiego 5B St., 02-106 Warsaw, Poland

E-mail: aziolk@ippt.gov.pl 
$\boldsymbol{A}_{\alpha}=\left\langle\breve{\boldsymbol{A}}_{\alpha}(\boldsymbol{x})\right\rangle_{V_{\alpha}} \equiv\left(1 / V_{\alpha}\right) \int_{V_{\alpha}} \breve{\boldsymbol{A}}(\boldsymbol{x}) \mathrm{d} x$ is an average of a local field over a region occupied by volume $V_{\alpha}$. When volume $V_{\alpha}$ is occupied by phase $\alpha$ then $\boldsymbol{A}_{\alpha}$ denotes $\alpha$ phase average.

It is accepted that internal energy and internal entropy of all kinds of martensitic phases at microscopic thermodynamic reference state (m.t.r.s.) - i.e., at zero local stresses $(\widetilde{\boldsymbol{\sigma}}(\boldsymbol{x})=0)$ and reference temperature $\left(T=T_{0}\right)$, are the same, e.g., $\left(u_{\alpha, \alpha \neq 1}^{0}=u_{\mathrm{M}_{\alpha}}=u_{A}-\Delta u^{0}, u_{A} \equiv u_{1}^{0}\right)$ and $\left(s_{\alpha, \alpha \neq 1}^{0}=s_{\mathrm{M}_{\alpha}}=s_{A}-\Delta s^{0}, s_{A} \equiv\right.$ $\left.s_{1}^{0}\right)$. The macroscopic thermodynamic reference state (t.r.s.) is a state at zero macroscopic stress $(\sigma=0)$ and reference temperature $\left(T=T_{0}\right)$.

$$
\boldsymbol{M}^{\mathrm{Re}} \equiv \sum_{\alpha=1}^{n} z_{\alpha} \boldsymbol{M}_{\alpha}, \quad \boldsymbol{L}^{V} \equiv \sum_{\alpha=1}^{n} z_{\alpha} \boldsymbol{L}_{\alpha}, \quad \boldsymbol{L}_{\alpha}=\boldsymbol{M}_{\alpha}^{-1}
$$

\section{Introduction}

It is well known that SMA elements of smart engineering structures under thermomechanical uniaxial and/or multiaxial loads undergo drastic microstructural changes which are associated with martensitic phase transition (p.t.). The martensitic phase transitions exhibit on the macroscopic scale in the form of a number of unique effects. They can be generally divided into the pseudoelasticity effects at higher temperatures $T$ and the oneway memory (quasi-plasticity) effects at lower temperatures. Surveys on behavior and thermodynamics of SMA materials including diverse modeling approaches can be found, e.g., in [3,9,10] or chapter 3 in [32]. A minute inspection of, e.g., [20] or [19], reveals that the determination of the macroscopic Gibbs free energy function $g$ for the representative volume element (RVE) requires specification of three fundamental terms: (a) the elastic complementary energy $g^{\text {el }}$ (or the effective elastic compliance, $\boldsymbol{M}$ ), (b) the coherence energy $\phi^{\text {coh }}-\mathrm{a}$ term coined by Müller and Seelecke [13] and (c) the macroscopic phase strain $\boldsymbol{\varepsilon}^{\mathrm{pt}}$ or the phase transformation work — bounded by the ultimate potential energy $g^{\text {ult }}$ of an external devise $-g^{\text {ult }} \equiv \sigma \cdot \varepsilon^{\mathrm{pt}}$.

The proper determination of these terms constitutes an open scientific problem of SMA thermodynamics, as the actual microstructure geometry evolution of representative volume element (RVE) under thermomechanical loadings (for multiaxial macroscopic stress histories $\sigma$ ) is in fact unknown. In the literature devoted to SMA modeling, specification of their explicit mathematical form is usually postulated ad hoc, separately for each term, entailing hard for estimation inaccuracies—cf., e.g., [9,22]. In order to improve this situation Ziolkowski and Raniecki [31] — cf. also chapter 4 in [32]—investigated a problem of micromechanics of two-phase RVE consisting of linear elastic phases with eigenstrains to be able to attribute terms present in Gibbs function to actual physical objects. Stress and strain fields were suitably subdivided to attribute them to various physical circumstances. An important outcome of this study has been realization of the fact that even after full completion of martensitic phase transition ( $z=1, z$-volume fraction of martensitic phase), in the single-phase SMA material there is usually accumulated considerable energy contribution resulting from phase's interaction during progress of martensitic phase transition. In the present work it is called stored coherency energy. In completely independent effort the issue of structure of macroscopic free energy of SMA material was addressed by Bernardini [2], who elaborated micromechanics-based method of the combined estimation of all three terms (a)-(c) for two-phase RVE. He took advantage of the fact that in the case of two-phase medium there are unique connections between fourth-order dimensionless mechanical concentration factor tensors, commonly denoted by the symbol $\boldsymbol{B}_{\alpha}$-cf., e.g., [5,6,8]—and other properly selected tensor measures of stress concentration. It can be shown that at fixed microstructure geometry and microscopic phase strain field, the tensors $\boldsymbol{B}_{\alpha}, \boldsymbol{\varepsilon}^{\mathrm{pt}}-(\mathrm{c})$ and scalar $\phi^{\text {coh }}$ - (b) become unique functions of $\boldsymbol{M}$. Estimate of $\boldsymbol{M}$ entails definite estimations of the terms (b) and (c). Thus, Bernardini [2] showed that for two-phase RVE the Gibbs free energy can be evaluated with the same level of approximation involved in the estimate of the effective elastic moduli. However, for multiphase medium comprising more than two phases $(n>2)$, unique connections between $\boldsymbol{B}_{\alpha}$ and $\boldsymbol{M}$ do not exist.

In the present paper elaborations presented in Bernardini, Ziolkowski and Raniecki [2,31] are further advanced among the others to verify the aptness of expressing the macroscopic coherence energy in the form of a quadratic function of phase composition - an ad hoc postulate in many papers devoted to SMA materials modeling. Attention is focused on micromechanics-based phenomenological modeling of properties of $n$ phase SMA material consisting of austenite $\mathrm{A}$ and $n-1$ generic martensitic objects $\mathrm{M}_{i}$. It is conjectured that the material can be in multiaxial stress states due to arbitrary single, half-cycle thermomechanical loading. The considerations are restricted to a class of uniform tractions (UT) boundary conditions prescribed on the boundary of RVE—not a critical constraint, assuming that the local fields of elastic compliance $\breve{M}(\boldsymbol{x})$ 
and transformation microstrains field $\breve{\boldsymbol{\Gamma}}(\boldsymbol{x})$ are piecewise uniform. This last conjecture is released for $\breve{\boldsymbol{\Gamma}}(\boldsymbol{x})$ field in Sect. 6. The results of classical homogenization theory presented in Dvorak and Benveniste, Dvorak [5,6] cf. also [2] are used in a unique modified manner which employs symmetric and skew-symmetric ingredients of self-equilibrated eigenstrains influence moduli (SEIM) to propose a new estimation method. The method served for presenting the terms (a) and (b) in the form of semilinear and/or semiquadratic functions of phase composition. The coefficients of these functions are expressed in terms of ingredients of SEIM tensors. The SEIM tensors are not independent as they must satisfy definite consistency conditions following from elastic reciprocity (Betti) theorem. A novel semiexperimental method for assessment of effective thermostatic properties of $n$-phase SMA macroelement is proposed. Application of the semiexperimental method to data for NiTi alloy revealed that the elastic heterogeneity in an isotropic mixture of two isotropic phases is of secondary importance in the evaluation of coherence energy $\phi^{\text {coh }}$. It is exposed that when microeigenstrains field is admitted which is not piecewise uniform, then in the martensitic phase an energy term arises resulting from phases interaction during p.t. progress, which still exists in pure martensite after phase transition is fully completed. This stored coherency energy contribution is connected with nonzero fluctuating part of phase microeigenstrains fields. Experimental data for NiTi alloy show that the stored coherency energy cannot be neglected as it considerably influences the characteristic phase transition temperatures of SMA material.

The paper is edited as follows. In Sect. 2 contemporary apparatus of nonequilibrium thermodynamics used in modeling of SMA materials behavior is shortly outlined. Subsequently adopted in the paper basic assumptions concerning the properties of individual phases at microscale are specified together with resulting from it mathematically precise definitions of the macroscopic complementary elastic energy, phase strain and coherency energy. In Sect. 3 development of original method for assessment of effective (macroscopic) thermostatic properties well suited for SMA materials is elaborated based on so-called self-equilibrated eigenstrains influence moduli. In Sect. 4 the method is applied to obtain consistent estimates of SMA material Gibbs function elements under classical assumptions of Reuss and Voigt. In Sect. 5 special engineering practice-oriented variant of SEIM estimation method is proposed. Next, explicit estimates of thermostatic properties of SMA material are obtained for several specific cases of possible material symmetries, homogeneity of appearing microstructures and/or distinguished number of phases. In Sect. 6 adopted in previous sections assumption on piecewise homogeneity of phase microeigenstrain field is released, and it is studied how fluctuating part of phase eigenstrains influences coherency energy term.

\section{Thermodynamic and micromechanical settings}

\subsection{Gibbs free energy function}

In contemporary works devoted to development of phenomenological model of SMA materials behavior it is commonly accepted at present that representative volume element (RVE) of SMA material makes an open thermodynamic system, which in prevailing time moments remains in the state of constrained thermodynamic equilibrium. An apparatus of nonequilibrium thermodynamics with internal state parameters has been developed for description of such systems, cf., e.g., [7]. Typically a macroelement state is described with external state parameters: temperature and stress $(\sigma, T)$, or temperature and strain $(\varepsilon, T)$, and a number of so-called internal (hidden) state parameters: such as, e.g., a set of parameters $h$ describing geometrical evolution of RVE microstructure. The hidden parameters can be of various tensorial characters, e.g., macroscopic ultimate phase eigenstrains $\boldsymbol{\kappa}$ appearing in definition of macroscopic phase eigenstrains $\boldsymbol{\varepsilon}^{\mathrm{pt}}$ is a second-order tensor $\left(\varepsilon^{\mathrm{pt}}=z \kappa\right)$; scalar $z$ denotes volume fraction of martensitic phase (objects). Usually at present for ultimate phase eigenstrains tensor some evolution law is proposed what effectively results in functional dependence of $\kappa$ on external state parameters $(\kappa=\kappa(\sigma, T))$. The most common at present conjecture on SMA materials RVE is that it consists of two phases: austenite A (phase no. 1) instantaneously occupying volume $V_{1}$ in total volume $V$ of the RVE and martensite M (phase no. 2) by which it is understood complex microstructure of all martensitic objects occupying remaining volume of the RVE. The volume fraction of $\mathrm{A}$ is $z_{1}=V_{1} / V$, and the volume fraction of martensite is $1-V_{1} / V=1-z_{1}=z_{2}=z$. The generic phases are treated as ideal inviscid, linear thermoelastic solids, perfectly bounded at their interfaces and undergoing small displacement gradients (there is no defect in the microstructure; no slip-like plastic flow accompanies the process of deformation, e.g., the viscosity of a material is neglected). It is usually accepted that mass density $\rho=\rho_{1}=\rho_{2}$ of both phases is at all instants the same. The specific heats (at constant stress) $c_{p}$ and thermal expansion coefficient $\boldsymbol{\alpha}$ are assumed to be the same and constant. The temperature dependence of these properties including effective 
(macroscopic) elastic stiffness moduli $\boldsymbol{L}$ and elastic compliances $\boldsymbol{M}=\boldsymbol{L}^{-1}$ is also typically neglected. The forward or reverse martensitic phase transition proceeds by motion of phase interfaces. It is the basic source of the energy dissipation which, on the macroscopic level, is exhibited in the form of various thermomechanical hysteresis loops-cf., e.g., [1,21]. In the present paper thermostatic equilibrium states of RVE are studied appearing at specific instants of SMA macroelement loading-unloading processes. They can be best imagined as frozen states at specific constant values of external parameters of stress and temperature- thus all internal state variables also have fixed values. It is usually accepted that SMA RVE is in thermal and mechanical equilibrium at every instant of active phase transition. The issues connected with phase transition processes, criteria for their initiation, and associated with them dissipative processes in relation to specific form of SMA material free energy will be broader discussed in a separate work. Under aforementioned simplifications the macroscopic specific Gibbs free energy function $g$ is frequently adopted in the following form—cf. [20,22,23],

$$
\begin{aligned}
g(\boldsymbol{\sigma}, T, z) & =\left(u_{1}^{0}-T s_{1}^{0}\right)-z \pi_{0}^{f}(T)+c_{p}\left[\Delta T-T \ln \left(T / T_{0}\right)\right]-g^{\mathrm{el}}-\boldsymbol{\sigma} \cdot \boldsymbol{\varepsilon}^{\mathrm{th}} / \rho-\boldsymbol{\sigma} \cdot \boldsymbol{\varepsilon}^{\mathrm{pt}} / \rho+\phi^{\mathrm{coh}}, \\
\pi_{0}^{f}(T) & \equiv \Delta u^{0}-T \Delta s^{0}, \Delta u^{0}=u_{1}^{0}-u_{2}^{0}, \Delta s^{0}=s_{1}^{0}-s_{2}^{0}, \Delta T=\left(T-T_{0}\right), g^{\mathrm{el}} \equiv \frac{1}{2} \boldsymbol{\sigma} \cdot \boldsymbol{M} \boldsymbol{\sigma} / \rho, \\
\boldsymbol{\varepsilon}^{\mathrm{th}} & \equiv \boldsymbol{\alpha} \Delta T, \boldsymbol{\varepsilon}^{\mathrm{pt}}=z \boldsymbol{\kappa}, \phi^{\mathrm{coh}}=\phi_{2}^{\mathrm{st}} z_{2}+\phi_{i t} z_{1} z_{2}, \phi_{i t}=\phi_{12}-T s_{12} ; \phi_{2}^{\mathrm{st}}=\Delta u_{2}^{\mathrm{st}}-T \Delta s_{2}^{\mathrm{st}} .
\end{aligned}
$$

where $u_{\alpha}^{0}-T_{0} s_{\alpha}^{0}$ denotes free energy of austenitic and martensitic phase, respectively $(\alpha=1,2)$, at some conventional macroscopic thermodynamic reference state (t.r.s), i.e., at reference temperature $T=T_{0}$ and zero macroscopic stress $\sigma=0$. The term $g^{\text {el }}$ denotes elastic complementary energy, and tensor of effective (macroscopic) elastic compliance moduli $\boldsymbol{M}$ is assumed temperature-independent. The $\boldsymbol{\varepsilon}^{\text {th }}$ denotes thermal strains. The term $\sigma \cdot \varepsilon^{\mathrm{pt}} / \rho$ represents the phase transformation work. The $\phi^{\text {coh }}$ denotes nonnegative quantity termed here coherency energy. This energy is composed of energy of internal interactions $\phi_{i t} z_{1} z_{2}$ and stored energy of coherency $\phi_{2}^{\text {st }} z$, i.e., energy stored in SMA macroelement after full completion of martensitic phase transformation $(z=1)$. The $\Delta u_{2}^{\text {st }}, \Delta s_{2}^{\text {st }}, \phi_{12}, s_{12}$ are treated as material constants. In the SMA literature $\phi^{\text {coh }}$ is also called the energy of transformation microstrain's incompatibilities, the configurational energy, the misfit energy, the phase's interaction energy or the mixing energy.

Under fixed $\boldsymbol{M}, \boldsymbol{\varepsilon}^{\mathrm{pt}}, \phi^{\mathrm{coh}}$ and $z_{1}$, the macroscopic Gibbs function plays a role of thermodynamic potential for the total macroscopic strain and the specific entropy when expressed in terms of its basic canonical state variables $\{T, \sigma\}$. Thus two fundamental macroscopic thermal equations of state for macroscopic total strain $\boldsymbol{\varepsilon}$ and for macroscopic specific entropy $s$ consistent with (1) take the form

$$
\begin{aligned}
\boldsymbol{\varepsilon} & =-\rho \frac{\partial g}{\partial \boldsymbol{\sigma}}=\boldsymbol{M} \boldsymbol{\sigma}+\boldsymbol{\alpha} \Delta T+\boldsymbol{\varepsilon}^{\mathrm{pt}}, s=-\frac{\partial g}{\partial T}=s_{1}^{0}+c_{p} \ln \left(T / T_{0}\right)-\left(1-z_{1}\right) \Delta s^{0}+\boldsymbol{\alpha} \cdot \boldsymbol{\sigma} / \rho \\
\boldsymbol{\varepsilon}^{\mathrm{e}} & \equiv \boldsymbol{\varepsilon}-\boldsymbol{\varepsilon}^{\mathrm{th}}-\boldsymbol{\varepsilon}^{\mathrm{pt}}, \quad \boldsymbol{\sigma}=\boldsymbol{L} \boldsymbol{\varepsilon}^{\mathrm{e}} .
\end{aligned}
$$

where $\varepsilon^{\mathrm{e}}$ denotes macroscopic elastic strain. The reference temperature $T_{0}$ is usually assumed to be greater than the conventional austenite-finish temperature $A_{f}^{0}$, and austenitic phase ultimate eigenstrains are adopted to be zero $\left(\boldsymbol{\Gamma}_{1}=0\right)$. Hence, in the thermodynamic reference state (t.r.s.) an RVE is in the pure austenitic state $\left(z_{1}=1\right)$, and $T=T_{0}, \boldsymbol{\sigma}=0, s=s_{1}^{0}, u=u_{1}^{0}$, and macroscopic phase strain $\boldsymbol{\varepsilon}^{\mathrm{pt}}=0$. The shape and the orientation in space of RVE in (t.r.s.) are then regarded as the reference configuration for the definition of a macroscopic total strain $\varepsilon$. It is worth noting here that thermal equations of state for the macroscopic total strain and macroscopic specific entropy, with the same resulting mathematical form, are generated either by using the average of local field of the Gibbs free energy over the total volume of $R V E\left(g=\langle g(\boldsymbol{x})\rangle_{V_{R V E}}\right)$ as thermodynamic potential or by volume averaging of the corresponding local equations of state.

The experimental data processing consistent with the family of $R_{\mathrm{L}}$ models of pseudoelasticity for NiTi alloy were discussed in Raniecki et al. [24]. Detailed discussion on procedure for identification of the $R_{\mathrm{L}}$ model material parameters can be found in section 5.4 of Ziolkowski [32] together with material data identified for $\mathrm{Ni}_{51 \mathrm{at}} \%-\mathrm{Ti}$ alloy, cf. Table $5.5 \mathrm{ibid}$. A rough estimates of some terms occurring in (1) for NiTi alloys exhibiting good pseudoelastic behavior are as follows

$$
\begin{aligned}
& \rho \Delta u^{0}=\rho\left(u_{1}^{0}-u_{2}^{0}\right) \approx 100-200[\mathrm{MPa}], \quad \rho \Delta s^{0} \approx 0.3-0.6[\mathrm{MPa} / \mathrm{K}], \\
& \rho \phi^{\mathrm{coh}} \approx 1-4[\mathrm{MPa}], \quad\left|\boldsymbol{\sigma} \cdot \boldsymbol{\varepsilon}^{\mathrm{pt}}\right| \approx 10-20[\mathrm{MPa}], \quad \rho g^{\mathrm{el}}=\frac{1}{2} \boldsymbol{\sigma} \cdot \boldsymbol{M} \boldsymbol{\sigma} \approx 0.3-1.2[\mathrm{MPa}]
\end{aligned}
$$

The data (3) 4,5 were estimated for the material at stress level equal to about 200 [MPa]. The above terms depict the overall scenery for a phase transition behavior of the material. The chemical equilibrium temperature $\left(T_{\mathrm{eq}}=\right.$ 


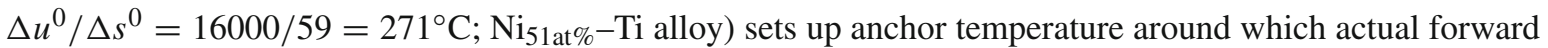
and reverse phase transitions occur depending on the actual value of the ratio between the macroscopic elastic strain energy $g^{\mathrm{el}}$ and the coherency energy $\phi^{\text {coh }}$, which both also enter into expression for a thermodynamic driving force of phase transition

$$
\pi^{1-2}(\boldsymbol{\sigma}, T, z ; \boldsymbol{\kappa}) \equiv \partial g / \partial z=\left(\pi_{0}^{f}-\phi_{2}^{\mathrm{st}}\right)-(1-2 z) \phi_{i t}+\boldsymbol{\kappa} \cdot \boldsymbol{\sigma} / \rho
$$

The basic problem of phenomenological thermodynamics of SMA material concerns the determination of the least number of physically plausible independent state parameters which represent the current rearrangement of internal microstructure, as well as the determination of specific dependence of $g^{\mathrm{el}}, \phi^{\mathrm{coh}}$ and $\varepsilon^{\mathrm{pt}}$ on those parameters. It still remains an open scientific problem. In order to provide more explicit the dependence of complementary elastic energy, coherency energy and transition strains on macroscopic state variables, at fixed geometry of microstructure, it is instrumental to submit to scrutiny the structure of macroscopic thermodynamic functions taking into account information on microscopic/metallurgical features of SMA materials behavior. Let us list several key characteristics of the dilemma. There have to be consistently estimated properties and/or variables of different tensorial order starting from scalars and ending on fourth-order tensors. Different number of RVE microconstituent modeling objects has to be distinguished depending on the complications of the microstructures that are to be modeled. Here, one component (chemically) but multiphase RVE aggregates are investigated, starting from two and ending on $n$ phases. First the problem of micromechanics is formulated for the most general heterogeneous case to be later simplified to piecewise constant fields of properties/variables with possible phase fluctuations. Important factor to be taken into account is symmetry resulting from RVE components or microstructure symmetries. Again first the most general case of nonisotropic RVE aggregate composed of nonisotropic components is investigated to end up on isotropic RVE aggregate composed of isotropic components. A vital factor worth careful attention is that phase eigenstrains are born and evolve under varying stresses, a situation very much resembling growth of soft tissues in biological organisms at presence of mechanical loadings-cf., e.g., Volokh [28]. The obvious consequence is that one cannot expect appearance of very ideal martensitic microstructures, like, e.g., regular plies in manmade composites. Fluctuations from average values have to be expected in the case of phase eigenstrains field. The question arises what is the influence of such irregularities on shape memory effects. It is assumed that arising during phase transitions displacements field is always continuous - consistently with property of coherency of martensitic transition (no cracks are generated in the SMA material).

2.2 Multiscale modeling of properties and state of martensitic structures: effective state variables and effective material properties

Let us accept as a starting point for the present study that in microscale of observation SMA material RVE occupying volume $V$ can be treated as a heterogeneous linear, thermoelastic body with eigenstrains, and that it obeys local constitutive relations of the form

$$
\breve{\boldsymbol{\varepsilon}}(\boldsymbol{x})=\breve{\boldsymbol{M}}(\boldsymbol{x}) \breve{\boldsymbol{\sigma}}(\boldsymbol{x})+\breve{\boldsymbol{\Gamma}}(\boldsymbol{x})+\boldsymbol{\alpha} \Delta T, \quad \Delta T=\left(T-T_{0}\right)
$$

All local fields will be denoted by conventional symbols with over-imposed check sign, and their dependence on time-like variable will not be here marked explicitly. Possible heterogeneity and temperature dependence of mass density $\rho$, specific heat, $c_{p}$ and thermal expansion coefficient $\alpha$ are neglected. Local elastic stiffness moduli $\breve{L}(\boldsymbol{x})$ and the local elastic compliances $\breve{\boldsymbol{M}}(\boldsymbol{x})=\breve{\boldsymbol{L}}^{-1}(\boldsymbol{x})$ fields are assumed to be independent on temperature. These assumptions are compatible with those usually made in macroscopic SMA material models.

Symbol $\breve{\boldsymbol{\Gamma}}(\boldsymbol{x})$ denotes heterogeneous field of phase eigenstrains, which is induced in RVE as a result of martensitic phase transitions and which can evolve under variation of external thermomechanical loadings of stress and temperature. In general, the field $\breve{\boldsymbol{\Gamma}}(\boldsymbol{x})$ does not satisfy geometrical compatibility conditions within the domain $V$ occupied by RVE. The incompatibility of phase eigenstrains field is the actual physical source of nonzero value of coherency energy $\phi^{\text {coh }}$.

It is commonly accepted conjecture that at each generic instant an SMA macroelement (RVE) under operational load remains in thermal and mechanical equilibrium. It may remain in constrained thermodynamic equilibrium only with respect to internal state parameters, e.g., phase fraction of martensitic phase $z$. Hence, 
local field of strains, through relations (5), must always fulfill equations of static mechanical equilibrium $(\operatorname{div} \widetilde{\sigma}(\boldsymbol{x})=0)$ with boundary conditions corresponding to actual load. The linear thermomicromechanics problem investigated here can be thought of as the one corresponding to a certain frozen microstructure of SMA material RVE, remaining under fixed load of macroscopic stress and temperature $(\sigma, T)$ with zero inertia and body forces. The temperature field in RVE is assumed to be uniform $\breve{T}(\boldsymbol{x})=T$, and mechanical loading is conjectured in the form of uniform tractions (U.T.) boundary conditions-thus Hill's postulate is identically satisfied $\left(\langle\breve{\boldsymbol{\sigma}}(\boldsymbol{x}) \cdot \breve{\boldsymbol{\varepsilon}}(\boldsymbol{x})\rangle_{V}=\langle\breve{\boldsymbol{\sigma}}(\boldsymbol{x})\rangle_{V} \cdot\langle\breve{\boldsymbol{\varepsilon}}(\boldsymbol{x})\rangle_{V}\right)$. Here the symbol $\langle\cdot\rangle_{V}$ denotes the volume average of the indicated field over the total domain occupied by RVE. The local elastic moduli and phase eigenstrains field

$\breve{M}(\boldsymbol{x})$ and $\breve{\boldsymbol{\Gamma}}(\boldsymbol{x})$ are assumed to be fixed and known. The original, investigated thermostatic, boundary-value problem of micromechanics can be replaced with the two problems as follows

$$
\begin{aligned}
& \breve{\boldsymbol{\varepsilon}}^{(\mathrm{ex})}(\boldsymbol{x})=\breve{\boldsymbol{M}}(\boldsymbol{x}) \breve{\boldsymbol{\sigma}}^{(\mathrm{ex})}(\boldsymbol{x}), \quad \operatorname{div} \breve{\boldsymbol{\sigma}}^{(\mathrm{ex})}=0 \quad \boldsymbol{x} \in V, \quad \boldsymbol{t}^{(n)}(x)=\boldsymbol{\sigma} \boldsymbol{n}(\boldsymbol{x}) \quad \boldsymbol{x} \in \partial V, \\
& \breve{\boldsymbol{\varepsilon}}^{(\mathrm{in})}(\boldsymbol{x})=\breve{\boldsymbol{M}}(\boldsymbol{x}) \breve{\boldsymbol{\sigma}}^{(\mathrm{in})}(\boldsymbol{x})+\breve{\boldsymbol{\Gamma}}(\boldsymbol{x}), \quad \operatorname{div} \breve{\boldsymbol{\sigma}}^{(\mathrm{in})}=0 \quad \boldsymbol{x} \in V, \quad \boldsymbol{t}^{(n)(\mathrm{in})}(\boldsymbol{x})=\breve{\boldsymbol{\sigma}}^{(\mathrm{in})}(\boldsymbol{x}) n=0 \quad \boldsymbol{x} \in \partial V
\end{aligned}
$$

The nonzero "external" stresses $\left(\sigma=\left\langle\breve{\boldsymbol{\sigma}}^{(\mathrm{ex})}(\boldsymbol{x})\right\rangle_{V}=\mathrm{const}\right)$ and strains in the first problem are caused by all-round surface tractions in the situation when $\breve{\boldsymbol{\Gamma}}(\boldsymbol{x})=0$, and in the second problem the "internal" stresses and strains are generated by incompatible $\breve{\boldsymbol{\Gamma}}(\boldsymbol{x})$ alone, at vanishing surface tractions. This operation enables to clearly distinguish the material response to surface tractions from that generated by eigenstrains. The field $\boldsymbol{\alpha} \Delta T$ has no influence on $\breve{\boldsymbol{\sigma}}^{\text {(ex) }}(\boldsymbol{x})$ in view of assumed uniformity of temperature field in RVE, whereas $\breve{\boldsymbol{\sigma}}^{\text {(in) }}(\boldsymbol{x})$ is a self-equilibrated stress field. As remarked in Bernardini [2], this kind of decomposition was used in former papers of Levin [11] and Rosen and Hashin [26].

The pair of total microstrain and microstress fields $\{\breve{\boldsymbol{\varepsilon}}(\boldsymbol{x}), \breve{\sigma}(\boldsymbol{x})\}$ solution to the original problem is simply a sum of the solutions of two auxiliary problems (6),

$$
\breve{\boldsymbol{\sigma}}(\boldsymbol{x})=\breve{\boldsymbol{\sigma}}^{(\mathrm{ex})}(\boldsymbol{x})+\breve{\boldsymbol{\sigma}}^{(\mathrm{in})}(\boldsymbol{x}), \quad \breve{\boldsymbol{\varepsilon}}(\boldsymbol{x})=\breve{\boldsymbol{\varepsilon}}^{(\mathrm{ex})}(\boldsymbol{x})+\breve{\boldsymbol{\varepsilon}}^{(\mathrm{in})}(\boldsymbol{x})+\boldsymbol{\alpha} \Delta T
$$

Experimental, metallurgical observations indicate that martensitic phase transformations are coherent, meaning that local microdisplacements field resulting from such transitions is continuous in the whole domain of SMA material RVE. In view of this property values of macroscopic (effective) stress and total strain $\boldsymbol{\sigma}, \boldsymbol{\varepsilon}$ (also partial stresses and strains computed, e.g., over volume of individual phases $\boldsymbol{\sigma}_{\alpha}, \boldsymbol{\varepsilon}_{\alpha}$ ) can be simply determined by averaging of local field values over volume of the RVE, respective partial volumes,

$$
\boldsymbol{\sigma}=\langle\breve{\boldsymbol{\sigma}}(\boldsymbol{x})\rangle_{V}=\left\langle\breve{\boldsymbol{\sigma}}(\boldsymbol{x})^{e x}\right\rangle_{V}, \quad \boldsymbol{\varepsilon}=\langle\breve{\boldsymbol{\varepsilon}}(\boldsymbol{x})\rangle_{V}=\left\langle\breve{\boldsymbol{\varepsilon}}(\boldsymbol{x})^{e x}\right\rangle_{V}, \quad \boldsymbol{\sigma}_{\alpha}=\langle\breve{\boldsymbol{\sigma}}(\boldsymbol{x})\rangle_{V_{\alpha}}, \quad \boldsymbol{\varepsilon}_{\alpha}=\langle\breve{\boldsymbol{\varepsilon}}(\boldsymbol{x})\rangle_{V_{\alpha}}
$$

Upon knowledge from some analytical studies, numerical computations or experimental measurements of local field solutions to (5)-(6) also elastic energy, energy of coherence of the macroelement and macroscopic phase strains can be determined by executing averaging procedure in accordance with the following formula — cf. also (1),

$$
\begin{aligned}
\rho g^{\mathrm{el}} & \equiv \frac{1}{2}\left\langle\breve{\boldsymbol{\sigma}}^{(\mathrm{ex})}(\boldsymbol{x}) \cdot \breve{\boldsymbol{M}}(\boldsymbol{x}) \breve{\boldsymbol{\sigma}}^{(\mathrm{ex})}(\boldsymbol{x})\right\rangle_{\mathrm{V}}=\frac{1}{2} \boldsymbol{\sigma} \cdot \boldsymbol{M} \boldsymbol{\sigma} ; \quad \boldsymbol{M}=\rho \partial^{2} g^{\mathrm{el}} / \partial \boldsymbol{\sigma} \partial \boldsymbol{\sigma} \\
\rho \phi^{\mathrm{coh}} & \equiv \frac{1}{2 V} \int_{V} \breve{\boldsymbol{\sigma}}^{(\mathrm{in})}(\boldsymbol{x}) \cdot \breve{\boldsymbol{M}}(\boldsymbol{x}) \breve{\boldsymbol{\sigma}}^{(\mathrm{in})}(\boldsymbol{x}) \mathrm{d} x=\frac{1}{2 V} \int_{V}\left(-\breve{\boldsymbol{\sigma}}^{(\mathrm{in})}(\boldsymbol{x})\right) \cdot \breve{\boldsymbol{\Gamma}}(\boldsymbol{x}) \mathrm{d} x \\
\boldsymbol{\varepsilon}^{\mathrm{pt}} & \equiv\left\langle\breve{\boldsymbol{\varepsilon}}^{(\mathrm{in})}(\boldsymbol{x})\right\rangle_{V}=\frac{1}{V} \int_{V}\left[\breve{\boldsymbol{\Gamma}}(\boldsymbol{x})+\breve{\boldsymbol{M}}(\boldsymbol{x}) \breve{\boldsymbol{\sigma}}^{(\mathrm{in})}(\boldsymbol{x})\right] \mathrm{d} x
\end{aligned}
$$

where $\boldsymbol{M}$ denotes effective compliance of SMA material RVE. The last expression in $(9)_{3}$ is obtained upon taking advantage of the property $\left\langle\overline{\boldsymbol{\sigma}}^{\text {(in) }}(\boldsymbol{x}) \cdot \breve{\boldsymbol{\varepsilon}}^{(\mathrm{in})}(\boldsymbol{x})\right\rangle_{V}=0$ resulting from the fact that $\breve{\boldsymbol{\sigma}}^{\text {(in) }}(\boldsymbol{x})$ is self-equilibrating field, cf. (6) 6 . It is straightforward to notice that when the local field $\breve{\boldsymbol{\Gamma}}(\boldsymbol{x})$ in the whole domain $(V)$ of RVE satisfies geometrical compatibility conditions then the self-equilibrated stress field $\breve{\sigma}^{\text {(in) }}$ and the coherency energy $\phi^{\text {coh }}$ are zero. The macroscopic elastic strain $\left(\boldsymbol{\varepsilon}^{\mathrm{e}}=\langle\breve{\boldsymbol{M}}(\boldsymbol{x}) \breve{\boldsymbol{\sigma}}(\boldsymbol{x})\rangle_{V}\right)$ should be carefully distinguished 
from the volume average of the local elastic strain field $\left\langle\widetilde{\boldsymbol{\varepsilon}}^{\mathrm{e}}(\boldsymbol{x})\right\rangle_{V}$. Upon averaging $(6)_{1}$ and taking advantage of $(9)_{3}$ it can be shown that $\left.\left\langle\widehat{\boldsymbol{\varepsilon}}^{\mathrm{e}}(\boldsymbol{x})\right\rangle_{V}=\boldsymbol{\varepsilon}^{\mathrm{e}}+\left\langle\breve{M}^{(\boldsymbol{x})}\right)^{\text {(in) }}(\boldsymbol{x})\right\rangle_{V}$.

The specific approach adopted in the present paper, aimed at obtaining sound estimates of effective (macroscopic) thermoelastic properties of SMA material, is by far not the only one possible. In order to locate the present development within the other contemporary approaches used for multiscale modeling of materials behavior and concerning homogenization methods interested reader can refer to a book by Guo [30].

\section{Self-equilibrated eigenstrains influence moduli (SEIM) method}

\subsection{Multiphase RVE with piecewise uniform elastic properties and microeigenstrain fields}

Microscopic observations on thermoelastic martensitic transformations, a phenomenon underlying shape memory effects in SMA materials, exhibit multiscale organization and features-see, e.g., Otsuka and Wayman [16] for more materials science biased information or Ziolkowski [32] for continuum mechanics biased one. Depending on the starting scale on which basic conjectures are made the objects to be distinguished can be single crystallographic variants of martensitic phase (CV), habit plane variants (HPV's) or, e.g., still higherorder structures like self-accommodating martensitic compounds, in the scale of observation where they can be meaningfully distinguished-cf., e.g., Nishida et al. [15]. A very versatile and comprehensive conjecture, which can be made to enable further modeling developments, is to accept that every single martensitic object or structure can be distinguished as a generic phase.

Let us now presuppose that the domain $V_{\mathrm{M}}$ of the complex martensitic microstructure can be subdivided into maximum $n-1$ subdomains of volumes $V_{\beta}$ with volume fractions $\mathrm{z}_{\beta}=V_{\beta} / V, \beta=2,3, \ldots, n,\left(1-z_{1}=\right.$ $\left.\sum_{\beta=2}^{n} \mathrm{z}_{\beta}\right)$. They are occupied by individual martensitic objects treated as a separate phases. This defines single-

component RVE being mixture of $n$ thermoelastic phases. The average $\boldsymbol{A}_{\alpha} \equiv\langle\breve{A}(\boldsymbol{x})\rangle_{V_{\alpha}}$ of any local field over a region occupied by the phase $\alpha,(\alpha=1,2, \ldots, n)$ will be further referred as the "phase average" of the field $\breve{\boldsymbol{A}}(\boldsymbol{x})$. The heterogeneous field of phase transition eigenstrains $\breve{\boldsymbol{\Gamma}}(\boldsymbol{x})$ can be now subdivided into an array of fields $\breve{\boldsymbol{\Gamma}}_{\beta}(\boldsymbol{x}), \boldsymbol{x} \in V_{\beta}(\beta=1,2,3, \ldots, n)$. Each $\breve{\boldsymbol{\Gamma}}_{\beta}(\boldsymbol{x})$ may be additively decomposed into phase average $\boldsymbol{\Gamma}_{\beta} \equiv\left\langle\breve{\boldsymbol{\Gamma}}_{\beta}(\boldsymbol{x})\right\rangle_{V_{\beta}}$ and phase fluctuation part $\breve{\boldsymbol{\Gamma}}_{\beta}^{f}(\boldsymbol{x})$ representing the deviation from the phase average. The latter quantity will be referred to as a phase fluctuating part of $\breve{\boldsymbol{\Gamma}}_{\beta}(\boldsymbol{x})$,

$$
\breve{\boldsymbol{\Gamma}}_{\beta}(\boldsymbol{x})=\boldsymbol{\Gamma}_{\beta}+\breve{\boldsymbol{\Gamma}}_{\beta}^{f}(\boldsymbol{x}), \quad \boldsymbol{\Gamma}_{\beta}=\left\langle\breve{\boldsymbol{\Gamma}}_{\beta}(\boldsymbol{x})\right\rangle_{V_{\beta}}
$$

Martensitic objects treated as separate phases are in general characterized by unequal elastic compliance tensor and micro-eigenstrains.

Let us accept at present further simplifying assumption that the fields $\breve{\boldsymbol{M}}(\boldsymbol{x})$ and $\breve{\boldsymbol{\Gamma}}(\boldsymbol{x})$ are piecewise uniform, and thus they are represented by arrays $\boldsymbol{M}_{\beta}$ and $\boldsymbol{\Gamma}_{\beta}$ of constant fourth-order and second-order symmetric tensors, respectively,

$$
\breve{\boldsymbol{M}}_{\beta}(\boldsymbol{x})=\boldsymbol{M}_{\beta}, \quad \breve{\boldsymbol{M}}_{\beta}^{f}(\boldsymbol{x})=0, \quad \breve{\boldsymbol{\Gamma}}_{\beta}(\boldsymbol{x})=\boldsymbol{\Gamma}_{\beta}, \quad \breve{\boldsymbol{\Gamma}}_{\beta}^{f}(\boldsymbol{x})=0, \quad \boldsymbol{x} \in V_{\beta}(\beta=1,2, \ldots, n) .
$$

The austenite shape is frequently taken as reference one, i.e., eigenstrain in the austenitic phase is accepted to be zero $\left(\boldsymbol{\Gamma}_{1}=0\right)$.

All the results presented in the remainder of Sects. 3 and 4 rely heavily on the conjectures (11). However, experimental data indicate that fluctuating parts of eigenstrains field $\breve{\boldsymbol{\Gamma}}_{\beta}(\boldsymbol{x})$ play considerable role in coherency energy. The modification of basic relations accounting for $\breve{\boldsymbol{\Gamma}}_{\beta}^{f}(\boldsymbol{x}) \neq 0$ is discussed in Sect. 6.

The local constitutive relations (5) with subdivision into external and internal fields (7) using conjecture (11) can now be written for local volume $V_{\alpha}$ of each phase as follows (! $\left.\alpha\right)$

$$
\begin{aligned}
\breve{\boldsymbol{\sigma}}_{\alpha}(\boldsymbol{x}) & =\breve{\boldsymbol{\sigma}}_{\alpha}^{(\mathrm{ex})}(\boldsymbol{x})+\breve{\boldsymbol{\sigma}}_{\alpha}^{(\mathrm{in})}(\boldsymbol{x}), \quad \breve{\boldsymbol{\varepsilon}}_{\alpha}(\boldsymbol{x})=\breve{\boldsymbol{\varepsilon}}_{\alpha}^{(\mathrm{ex})}(\boldsymbol{x})+\breve{\boldsymbol{\varepsilon}}_{\alpha}^{(\mathrm{in})}(\boldsymbol{x})+\boldsymbol{\alpha} \Delta T, \\
\breve{\boldsymbol{\varepsilon}}_{\alpha}^{(\mathrm{ex})}(\boldsymbol{x}) & =\boldsymbol{M}_{\alpha} \breve{\boldsymbol{\sigma}}_{\alpha}^{(\mathrm{ex})}(\boldsymbol{x}), \quad \breve{\boldsymbol{\varepsilon}}_{\alpha}^{(\mathrm{in})}(\boldsymbol{x})=\boldsymbol{M}_{\alpha} \breve{\boldsymbol{\sigma}}_{\alpha}^{(\mathrm{in})}(\boldsymbol{x})+\boldsymbol{\Gamma}_{\alpha}, \\
\breve{\boldsymbol{\varepsilon}}_{\alpha}(\boldsymbol{x}) & =\boldsymbol{M}_{\alpha} \breve{\boldsymbol{\sigma}}_{\alpha}(\boldsymbol{x})+\boldsymbol{\Gamma}_{\alpha}+\boldsymbol{\alpha} \Delta T, \quad \boldsymbol{x} \in V_{\alpha} .
\end{aligned}
$$


Eigenstrains field $\breve{\boldsymbol{\Gamma}}(\boldsymbol{x}) ;\left(\boldsymbol{\Gamma}_{\alpha}, \boldsymbol{x} \in V_{\alpha}\right)$ in general does not satisfy geometrical compatibility conditions at most of the interphases, where it usually undergoes jump discontinuities. Due to that local microstrain $\widetilde{\boldsymbol{\varepsilon}}_{\alpha}(\boldsymbol{x})$ and microstress $\widetilde{\sigma}_{\alpha}(\boldsymbol{x})$ fields may also be discontinuous at the interfaces between austenite and martensitic phases.

In further discussion devoted to overall thermoelastic properties of $n$-phase SMA materials RVE it is developed original approach based on self-equilibrated eigenstrains influence moduli (SEIM). It was motivated by the method developed in Dvorak and Benveniste [5], see also [6]. Similar approach for two-phase RVE of SMA materials was used in Bernardini, Bernardini and Masiani $[2,4]$.

3.2 Structure of the matrix of self-equilibrated eigenstrains influence moduli consistent with the elastic reciprocity theorem

Formal solution of mechanical equilibrium problems (6) for piecewise uniform elastostatic properties $\boldsymbol{M}_{\beta}$ and eigenstrains field $\boldsymbol{\Gamma}_{\beta}\left(\breve{\boldsymbol{\Gamma}}_{\alpha}^{f}(\boldsymbol{x}) \equiv 0\right)$-cf. conjectures (11), can be expressed with the aid of self-equilibrated eigenstrains influence moduli as follows,

$$
\breve{\boldsymbol{\sigma}}_{\alpha}^{(\mathrm{in})}(\boldsymbol{x})=\sum_{\beta=1}^{n} \widetilde{\Im}_{\alpha \beta}(\boldsymbol{x}) \boldsymbol{\Gamma}_{\beta}, \quad \breve{\boldsymbol{\sigma}}_{\alpha}^{(\mathrm{ex})}(\boldsymbol{x})=\boldsymbol{\sigma}+\sum_{\beta=1}^{n} \widetilde{\Im}_{\alpha \beta}(\boldsymbol{x}) \boldsymbol{M}_{\beta} \boldsymbol{\sigma}, \quad \boldsymbol{x} \in V_{\alpha},(\alpha, \beta=1,2 \ldots \ldots n)
$$

The local fields of external phase stresses $\breve{\boldsymbol{\sigma}}_{\alpha}^{(\mathrm{ex})}(\boldsymbol{x})$ are linear functions of quasi-strains $\boldsymbol{M}_{\beta} \boldsymbol{\sigma}$. The local internal phase stresses $\breve{\boldsymbol{\sigma}}_{\alpha}^{(\mathrm{in})}(\boldsymbol{x})$ are linear functions of all eigenstrains $\boldsymbol{\Gamma}_{\beta}$ and are self-equilibrated stresses

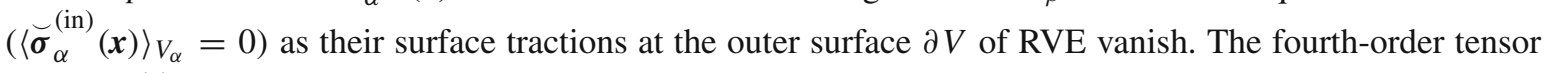
functions $\widetilde{\Im}_{\alpha \beta}(\boldsymbol{x})$ are the elements of $n \times n$ influence matrix $[\bullet]_{\alpha \beta}$. They are assumed to be known for prescribed geometry of the RVE microstructure. The mathematically rigorous formulas representing $\breve{\Im}_{\alpha \beta}(x)$ should assure $\breve{\boldsymbol{\sigma}}_{\alpha}^{\text {(in) }}(\boldsymbol{x})=0$ when the local eigenstrains field $\breve{\boldsymbol{\Gamma}}(\boldsymbol{x})$ is compatible $\left(\breve{\boldsymbol{\Gamma}}(\boldsymbol{x})=\boldsymbol{\Gamma}_{\beta}\right.$, for $\left.\in V_{\beta}\right)$. Their actual determination requires the separate laborious numerical evaluations based on methods discussed, e.g., in $[2,14,29]$ or [12]. The phase averages of $\widetilde{\Im}_{\alpha \beta}(\boldsymbol{x})$ are defined as follows

$$
\mathfrak{\Im}_{\alpha \beta}^{*} \equiv\left\langle\widetilde{\Im}_{\alpha \beta}(\boldsymbol{x})\right\rangle_{V_{\alpha}}=\left(1 / V_{\alpha}\right) \int_{V_{\alpha}} \widetilde{\Im}_{\alpha \beta}(\boldsymbol{x}) \mathrm{d} x
$$

We will call the fourth-order tensors $\mathfrak{\Im}_{\alpha \beta}^{*}$ self-equilibrated eigenstrains influence moduli. This type of tensors in the field of SMA materials modeling was introduced by Bernardini to the best knowledge of the present author. Upon comparing formula (26) in Bernardini [2] and formula (15) 1 the following correspondence in notation can be noticed $\Im_{\alpha \beta}^{*} \leftrightarrow F_{\alpha \beta}$.

Apart from $\Im_{\alpha \beta}^{*}$, the local field and its phase average will be denoted by the same kernel symbol. The phase average will be stripped off superimposed check sign. The phase averages of internal and external stresses are $(\boldsymbol{\alpha}, \beta=1,2, \ldots, n)$,

$$
\boldsymbol{\sigma}_{\alpha}^{(\mathrm{in})} \equiv\left\langle\breve{\boldsymbol{\sigma}}_{\alpha}^{(\mathrm{in})}(\boldsymbol{x})\right\rangle_{V_{\alpha}}=\sum_{\beta=1}^{n} \mathfrak{\Im}_{\alpha \beta}^{*} \boldsymbol{\Gamma}_{\beta}, \quad \boldsymbol{\sigma}_{\alpha}^{(\mathrm{ex})} \equiv\left\langle\breve{\boldsymbol{\sigma}}_{\alpha}^{(\mathrm{ex})}(\boldsymbol{x})\right\rangle_{V_{\alpha}}=\boldsymbol{\sigma}+\mathfrak{\Im}_{\alpha \beta}^{*} \boldsymbol{M}_{\beta} \boldsymbol{\sigma} .
$$

The fourth-order tensors of eigenstrains influence moduli $\mathfrak{\Im}_{\alpha \beta}^{*}$ are in general diagonally nonsymmetric tensors, and they are not independent of each other. Such nonsymmetry finds its roots in admissible nonsymmetry of microstructural patterns. By taking into account that $\widetilde{\Im}_{\alpha \beta}(\boldsymbol{x})$ must vanish when all $\boldsymbol{\Gamma}_{\alpha}$ coincide, and by invoking the Betti's elastic reciprocal theorem, it can be shown that $\Im_{\alpha \beta}^{*}$ must fulfill the following conditions $(\alpha, \beta=1,2, \ldots, n)$,

$$
\sum_{\alpha=1}^{n} \mathfrak{\Im}_{\beta \alpha}^{*}=\sum_{\alpha=1}^{n} \mathfrak{\Im}_{\beta \alpha}^{\mathrm{T}}=0, \quad z_{\alpha} \mathfrak{\Im}_{\alpha \beta}^{*}=z_{\beta} \mathfrak{\Im}_{\beta \alpha}^{\mathrm{T}},(! \alpha, ! \beta)
$$


The mathematical proof of specific form of constraints $(16)_{2}$ imposed by reciprocal (Betti) theorem can be found, e.g., in chapter 3 of Dvorak [6]. Care must be exercised to distinguish between symmetry of various objects. For example an influence matrix having as its components SEIM tensors $\mathfrak{\Im}_{\alpha \beta}^{*}$ would be symmetric when $\mathfrak{\Im}_{\alpha \beta}^{*}=\mathfrak{\Im}_{\beta \alpha}^{*} \quad(\alpha, \beta=1,2, \ldots, n)$. On the other hand diagonal symmetry of specific element of influence matrix say $\mathfrak{\Im}_{\mathrm{A} B}^{*}$, i.e., SEIM tensor itself, requires that $\mathfrak{\Im}_{\mathrm{A} B}^{*}=\left(\Im_{A B}^{*}\right)^{\mathrm{T}}$. The relations (16) imply $\Sigma_{\alpha=1}^{n} z_{\alpha} \Im_{\alpha \beta}^{*}=0$. From $(16)_{2}$ and (9) it comes out that the self-equilibrated moduli of eigenstrains influence $\Im_{\alpha \alpha}^{*}$ must be diagonally symmetric, positive semidefinite, fourth-order tensors $\left(\mathfrak{\Im}_{\alpha \alpha}^{*}=\left(\mathfrak{\Im}_{\alpha \alpha}^{*}\right)^{\mathrm{T}}\right)(! \alpha)$. Moreover, $\mathfrak{\Im}_{\alpha \beta}^{*}=0$ for $z_{\beta}=0$ and $z_{\alpha} \neq 0,(\alpha \neq \beta)$. Therefore, in order to satisfy $(16)_{2}$ for $\alpha \neq \beta$ it is proposed to restrict considerations to a class of microstructure geometries for which the following relations hold

$$
\mathfrak{\Im}_{\alpha \beta}^{*}=z_{\beta} \mathfrak{\Im}_{\alpha \beta}, \quad \mathfrak{\Im}_{\alpha \beta}=\mathfrak{\Im}_{\alpha \beta}^{(S)}+\mathfrak{\Im}_{\alpha \beta}^{(A)}, \quad \mathfrak{\Im}_{\alpha \beta}=\left(\mathfrak{\Im}_{\beta \alpha}\right)^{\mathrm{T}}, \quad(! \beta) .
$$

The fourth-order tensors $\mathfrak{\Im}_{\alpha \beta}^{(S)}=\frac{1}{2}\left(\Im_{\alpha \beta}+\Im_{\beta \alpha}\right)=\Im_{\beta \alpha}^{(S)}$ make elements of symmetric matrix. Likewise the tensors $\Im_{\alpha \beta}^{(A)}=\frac{1}{2}\left(\Im_{\alpha \beta}-\Im_{\beta \alpha}\right)=-\Im_{\beta \alpha}^{(A)}$ make elements of the skew-symmetric matrix. Using (17) it may readily be shown that all elements $\Im_{\alpha \beta}^{(S)}$ of symmetric matrix are diagonally symmetric fourth-order tensors $\left(\left(\Im_{\alpha \beta}^{(S)}\right)_{m n i j}=\left(\mathfrak{\Im}_{\alpha \beta}^{(S)}\right)_{i j m n}\right)$, whereas all elements of $\mathfrak{\Im}_{\alpha \beta}^{(A)}$ are diagonally skew-symmetric tensors $\left(\left(\mathfrak{\Im}_{\alpha \beta}^{(A)}\right)_{m n i j}=\right.$ $\left.-\left(\mathfrak{\Im}_{\alpha \beta}^{(A)}\right)_{i j m n}\right)$,

$$
\mathfrak{\Im}_{\alpha \beta}^{(S)}=\mathfrak{\Im}_{\beta \alpha}^{(S)}, \mathfrak{\Im}_{\alpha \beta}^{(S)}=\mathfrak{\Im}_{\alpha \beta}^{\mathrm{T}}, \quad \mathfrak{\Im}_{\alpha \beta}^{(A)}=-\mathfrak{\Im}_{\beta \alpha}^{(A)}, \mathfrak{\Im}_{\alpha \beta}^{(A)}=\mathfrak{\Im}_{\beta \alpha}^{\mathrm{T}}, \quad \text { for } \alpha \neq \beta
$$

The first condition (16) 1 together with (17) provides the connections between symmetric self-induced moduli of eigenstrains influence $\Im_{\alpha \alpha}^{*}=\left(\Im_{\alpha \alpha}^{*}\right)^{\mathrm{T}}(! \alpha)$ and nondiagonal moduli $\mathfrak{\Im}_{\alpha \beta}^{(S)},(\alpha \neq \beta)$. They are not influenced by $\mathfrak{\Im}_{\alpha \beta}^{(A)}$, of course. The condition $(16)_{1}$ shows also that elements $\mathfrak{\Im}_{\alpha \beta}^{(A)}$ of skew-symmetric matrix are not independent,

$$
\mathfrak{\Im}_{\alpha \alpha}^{*}=-\sum_{\beta=1, \beta \neq \alpha}^{n} z_{\beta} \Im_{\alpha \beta}^{(S)}, \quad \sum_{\beta=1, \beta \neq \alpha}^{n} z_{\beta} \Im_{\alpha \beta}^{(A)}=-\sum_{\beta=1, \beta \neq \alpha}^{n} z_{\beta} \Im_{\beta \alpha}^{(A)}=0, \quad(! \alpha=1,2, \ldots, n)
$$

Thus, in general mathematically precise description of a state of macroelement (RVE) consisting of parent phase and $n-1$ martensitic phases requires appropriate specification of $\mathfrak{\Im}_{\alpha \beta}^{*}$ in terms of $n(n-1) / 2$ fourth-order symmetric tensors $\mathfrak{\Im}_{\alpha \beta}^{(S)}$ and $(n-2)(n-1) / 2>0$ fourth-order diagonally skew-symmetric tensors $\mathfrak{\Im}_{\alpha \beta}^{(A)}$. The skew-symmetric tensors $\mathfrak{\Im}_{\alpha \beta}^{(A)}$ are bound by $n$ constraint equations $(19)_{2}$ from which only $n-1$ is linearly independent. In other words, there are $n+(n-1)$ independent relations for the $(n \times n)$ unknown moduli $\mathfrak{\Im}_{\alpha \beta}^{*}$ of the self-equilibrated eigenstrains influence. For example: if $n=2$ then the knowledge of only one diagonally symmetric tensor is needed to determine four moduli $\mathfrak{\Im}_{\alpha \beta}^{*}\left[\Im_{\alpha \beta}^{(A)}=0\right.$ on account of (19)], if $n=3$ the three, fourth-order, diagonally symmetric tensors and one diagonally skew-symmetric tensor are needed to determine nine moduli $\mathfrak{\Im}_{\alpha \beta}^{*}$. The latter situation is generalization to $n$ phases of the result presented for $n=2$ in [2].

The general connection between $\mathfrak{\Im}_{\alpha \beta}^{*}$ and the couple $\left\{\mathfrak{\Im}_{\alpha \beta}^{(S)}, \mathfrak{\Im}_{\alpha \beta}^{(A)}\right\}$ may be written in the form involving $n$-dimensional Kronecker second-order symbol $\delta_{\alpha \beta}$,

$$
\mathfrak{\Im}_{\alpha \beta}^{*}=\left(1-\delta_{\alpha \beta}\right) z_{\beta}\left(\Im_{\alpha \beta}^{(S)}+\Im_{\alpha \beta}^{(A)}\right)-\delta_{\alpha \beta} \sum_{\gamma=1}^{n}\left(1-\delta_{\gamma \alpha}\right) z_{\gamma} \Im_{\alpha \gamma}^{(S)} \quad(! \alpha, \beta) .
$$

The tensors $\Im_{\alpha \beta}^{(S)}$ and $\Im_{\alpha \beta}^{(A)}$ will be collectively referred to as the ingredients of self-equilibrated eigenstrains influence moduli. In general, they do depend on phase composition $z_{\alpha}$, elastic moduli of individual phases $\boldsymbol{M}_{\alpha}$ and a number of descriptors of the microstructure geometry, but they are not influenced by $\boldsymbol{\Gamma}_{\beta}$.

As it will be shown in the next sections, it turns out that the terms $g_{\alpha \beta}^{\text {el }}$ and $\phi_{\alpha \beta}^{(0) c o h}-\mathrm{cf}$. (27) and (29) below, can be expressed in a useful and compact form provided the following family of scalar functions 
$\mathbf{f}_{\alpha \beta}(\boldsymbol{X}, \boldsymbol{Y}),(\alpha \neq \beta)$ of two second-order symmetric tensors $\boldsymbol{X}, \boldsymbol{Y}$ is introduced. The functions $\mathrm{f}_{\alpha \beta}$ are defined as follows,

$$
\begin{aligned}
& f_{\alpha \beta}(\boldsymbol{X}, \boldsymbol{Y}) \equiv 0.5\left[(\boldsymbol{X}-\boldsymbol{Y}) \cdot \Im_{\alpha \beta}^{(S)}(\boldsymbol{X}-\boldsymbol{Y})-\left(\boldsymbol{X} \cdot \Im_{\alpha \beta}^{(A)} \boldsymbol{Y}-\boldsymbol{Y} \cdot \Im_{\alpha \beta}^{(A)} \boldsymbol{X}\right)\right], \\
& f_{\alpha \beta}(\boldsymbol{X}, \boldsymbol{Y})=f_{\beta \alpha}(\boldsymbol{Y}, \boldsymbol{X}), \quad f_{\alpha \beta}(\boldsymbol{X}, \boldsymbol{X})=0 .
\end{aligned}
$$

Similarly phase stresses and strains can be expressed in compact form with an array $\mathfrak{R}_{\alpha}\left(\chi_{\gamma}\right)$ of linear secondorder tensor functions of $n$ second-order symmetric tensors $\chi_{\gamma},(\alpha, \gamma=1,2, \ldots, n)$,

$$
\mathfrak{\Re}_{\alpha}\left(\chi_{\gamma}\right) \equiv \sum_{\beta=1}^{n} \mathfrak{\Im}_{\alpha \beta}^{*} \chi_{\beta}, \quad \mathfrak{\Re}_{\alpha}\left(\chi_{\gamma}\right)=\sum_{\beta=1, \quad \beta \neq \alpha}^{n} z_{\beta}\left[\mathfrak{\Im}_{\alpha \beta}^{(S)}\left(\chi_{\beta}-\chi_{\alpha}\right)+\mathfrak{\Im}_{\alpha \beta}^{(A)} \boldsymbol{\chi}_{\beta}\right], \quad\left(\sum_{\alpha=1}^{n} z_{\alpha} \Re_{\alpha}=0\right),
$$

In general, the specific forms of both $\mathfrak{R}_{\alpha}$ and $f_{\alpha \beta}$ besides dependence on phase composition $z_{\gamma},(\gamma=$ $1,2, \ldots, n)$ depend parametrically on variables describing the microstructure of SMA macroelement. The following properties were used to obtain convenient form of functions $\Re_{\alpha}$ given in (22)

$$
\sum_{\alpha, \beta=1, \alpha \neq \beta}^{n} z_{\alpha} z_{\beta} \Im_{\alpha \beta}^{(S)}\left(\chi_{\beta}-\chi_{\alpha}\right)=0, \quad \sum_{\alpha, \beta=1, \alpha \neq \beta}^{n} z_{\alpha} z_{\beta} \Im_{\alpha \beta}^{(A)} \chi_{\beta}=0.5 \sum_{\alpha, \beta=1, \alpha \neq \beta}^{n} z_{\alpha} z_{\beta} \Im_{\alpha \beta}^{(A)}\left(\chi_{\beta}-\chi_{\alpha}\right)=0 .
$$

3.3 Description of macroscopic thermostatic properties in terms of self-equilibrated eigenstrains influence moduli

Standard micromechanical computations Dvorak [6] show that phase averages of internal, external and total microstresses - cf. (13), may thus be presented in terms of ingredients of self-equilibrated eigenstrains influence moduli $\mathfrak{\Im}_{\alpha \beta}^{*}(\alpha, \beta=1,2, \ldots, n)$ with the aid of tensor functions $\Re_{\alpha}$ defined in (22) as follows,

$$
\boldsymbol{\sigma}_{\alpha}^{(\mathrm{in})}=\mathfrak{\Re}_{\alpha}\left(\boldsymbol{\Gamma}_{\gamma}\right), \quad \boldsymbol{\sigma}_{\alpha}^{(\mathrm{ex})}=\boldsymbol{\sigma}+\mathfrak{\Re}_{\alpha}\left(\boldsymbol{M}_{\gamma} \boldsymbol{\sigma}\right), \quad \boldsymbol{\sigma}_{\alpha}=\boldsymbol{\sigma}+\mathfrak{R}_{\alpha}\left(\boldsymbol{M}_{\gamma} \boldsymbol{\sigma}+\boldsymbol{\Gamma}_{\gamma}\right) .
$$

Refer now to (12) and evaluate the phase averages of local strains in each phase domain,

$$
\boldsymbol{\varepsilon}_{\alpha}^{(\mathrm{ex})}=\boldsymbol{M}_{\alpha} \boldsymbol{\sigma}_{\alpha}^{(\mathrm{ex})}, \quad \boldsymbol{\varepsilon}_{\alpha}^{(\mathrm{in})}=\boldsymbol{M}_{\alpha} \boldsymbol{\sigma}_{\alpha}^{(\mathrm{in})}+\boldsymbol{\Gamma}_{\alpha}, \quad \boldsymbol{\varepsilon}_{\alpha}=\boldsymbol{M}_{\alpha} \boldsymbol{\sigma}_{\alpha}+\boldsymbol{\alpha} \Delta \boldsymbol{T}+\boldsymbol{\Gamma}_{\alpha} \quad(! \alpha) .
$$

By making use of (22), (23) and (24) the macroscopic phase strain $\varepsilon^{\mathrm{pt}}-\mathrm{cf} .(9)_{3}$, and the effective elastic compliance $\boldsymbol{M}$ occurring in (1) can be expressed in terms of $\Im_{\alpha \beta}^{(S)}$ and $\Im_{\alpha \beta}^{(A)}$ as follows

$$
\begin{aligned}
\boldsymbol{M} & =\boldsymbol{M}^{\mathrm{Re}}-\sum_{\alpha, \beta=1, \beta \neq \alpha}^{n} z_{\alpha} z_{\beta} \boldsymbol{M}_{\alpha \beta}, \quad \boldsymbol{M}^{\mathrm{Re}} \equiv \sum_{\alpha=1}^{n} z_{\alpha} \boldsymbol{M}_{\alpha}, \quad \Delta \boldsymbol{M}_{\alpha \beta} \equiv \boldsymbol{M}_{\alpha}-\boldsymbol{M}_{\beta}, \\
\boldsymbol{M}_{\alpha \beta} & =0.5\left[\Delta \boldsymbol{M}_{\alpha \beta} \Im_{\alpha \beta}^{(S)} \Delta \boldsymbol{M}_{\alpha \beta}-\left(\boldsymbol{M}_{\alpha} \Im_{\alpha \beta}^{(A)} \boldsymbol{M}_{\beta}-\boldsymbol{M}_{\beta} \Im_{\alpha \beta}^{(A)} \boldsymbol{M}_{\alpha}\right)\right], \quad \boldsymbol{M}_{\alpha \beta}=\boldsymbol{M}_{\beta \alpha}, \quad \boldsymbol{M}_{\alpha \beta}=\boldsymbol{M}_{\alpha \beta}^{\mathrm{T}} .
\end{aligned}
$$

and

$$
\boldsymbol{\varepsilon}^{(\mathrm{ex})}=\boldsymbol{M}^{\mathrm{Re}} \boldsymbol{\sigma}+\sum_{\alpha=1}^{n} z_{\alpha} \boldsymbol{M}_{\alpha} \Re_{\alpha}\left(\boldsymbol{M}_{\gamma} \boldsymbol{\sigma}\right)=M \boldsymbol{\sigma}, \quad \boldsymbol{\varepsilon}^{\mathrm{pt}}=\boldsymbol{\Gamma}+\sum_{\alpha=1}^{n} z_{\alpha} \boldsymbol{M}_{\alpha} \Re_{\alpha}\left(\boldsymbol{\Gamma}_{\gamma}\right), \boldsymbol{\Gamma}=\sum_{\alpha=1}^{n} z_{\alpha} \boldsymbol{\Gamma}_{\alpha} .
$$

Here $\boldsymbol{M}^{\mathrm{Re}}$ is the Reuss upper bound of the macroscopic (effective) elastic compliance $\boldsymbol{M}$.

Taking into account definition $(9)_{1}$, the macroscopic elastic complementary energy of the solid phase mixture $g^{\text {el }}$ can be now expressed in terms of $\Im_{\alpha \beta}^{(S)}$ and $\Im_{\alpha \beta}^{(A)}$ upon using (25),

$$
\begin{aligned}
g^{\mathrm{el}} & =\sum_{\alpha=1}^{n} z_{\alpha} g_{\alpha}^{(0) \mathrm{el}}-0.5 \sum_{\alpha, \beta=1, \alpha \neq \beta}^{n} z_{\alpha} z_{\beta} g_{\alpha \beta}^{\mathrm{el}}, \\
\rho g_{\alpha}^{(0) \mathrm{el}} & \equiv 0.5 \boldsymbol{\sigma} \cdot \boldsymbol{M}_{\alpha} \boldsymbol{\sigma}, \quad \rho g_{\alpha \beta}^{\mathrm{el}} \equiv \boldsymbol{\sigma} \cdot \boldsymbol{M}_{\alpha \beta} \boldsymbol{\sigma}=\mathrm{f}_{\alpha \beta}\left(\boldsymbol{M}_{\alpha} \boldsymbol{\sigma}, \boldsymbol{M}_{\beta} \boldsymbol{\sigma}\right) .
\end{aligned}
$$


The second term present in $(27)_{1}$ is semiquadratic form of phase composition as the ingredients $g_{\alpha \beta}^{\mathrm{el}}$ in general may also be influenced by phase volume fractions. It becomes the genuine quadratic form of phase composition, provided that both $\Im_{\alpha \beta}^{(A)}$ and $\Im_{\alpha \beta}^{(S)}$ are independent of $z_{\alpha}$.

Let us refer now to $(9)_{2}$. Since phase eigenstrains field is assumed to be piecewise uniform in RVE, cf. (11), the coherency energy becomes the following positive semidefinite quadratic function of phase eigenstrains $\boldsymbol{\Gamma}_{\alpha}$,

$$
\rho \phi^{\mathrm{coh}}=0.5 \sum_{\alpha=1}^{n} z_{\alpha}\left(-\boldsymbol{\sigma}_{\alpha}^{(\mathrm{in})}\right) \cdot \boldsymbol{\Gamma}_{\alpha} \Rightarrow \rho \phi^{\mathrm{coh}}=-0.5 \sum_{\alpha, \beta=1}^{n} z_{\alpha} \boldsymbol{\Gamma}_{\alpha} \cdot \Im_{\alpha \beta}^{*} \boldsymbol{\Gamma}_{\beta} \geq 0 .
$$

The above expressions are identical with the expressions (38) in Bernardini [2]. By substituting (20) into (28) after some algebraic transformations the second form of coherency energy may be rewritten in the form similar to the second term occurring in $(27)_{1}$,

$$
\phi^{\mathrm{coh}}=0.5 \sum_{\alpha, \beta=1, \alpha \neq \beta}^{n} z_{\alpha} z_{\beta} \phi_{\alpha \beta}^{(0) \mathrm{coh}}, \quad \rho \phi_{\alpha \beta}^{(0) \mathrm{coh}}=\mathrm{f}_{\alpha \beta}\left(\boldsymbol{\Gamma}_{\alpha}, \boldsymbol{\Gamma}_{\beta}\right) .
$$

Note that $\phi_{\alpha \beta}^{(0) \text { coh }}=\phi_{\beta \alpha}^{(0) \text { coh }}$ are independent of $\Im_{\alpha \beta}^{(A)}$ provided that $\boldsymbol{\Gamma}_{\alpha}=0$. This follows directly from the definition of $\mathrm{f}_{\alpha \beta}-\mathrm{cf}$. (21). The physical interpretation of the coherence coefficients $\phi_{\alpha \beta}^{(0) \text { coh }}$ basing on (28) 1 is that they describe partial work contribution of microstresses generated by uniform phase eigenstrains of phase $\beta$ done on uniform eigenstrains of phase $\boldsymbol{\alpha}$. Please note that (29) is valid only when $\breve{\boldsymbol{\Gamma}}_{\beta}^{f}(\boldsymbol{x})=0$.

\subsubsection{Case of nonisotropic two-phase elastic media forming nonisotropic aggregate}

By far the most widespread assumption made in modeling literature on SMA materials is conjecture that it is a two-phase mixture of austenite and martensite. Due to that for elucidation purposes relations developed in previous section for estimates of phase eigenstrains, elastic and coherency energies are written explicitly for this case. For two-phase media $(n=2)$ condition $(19)_{2}$ implies $\Im_{\alpha \beta}^{(A)}=0$. Hence, all self-equilibrated eigenstrains influence moduli appearing in general relation (20), fulfilling the requirements (16), can be expressed in terms of a single, diagonally symmetric tensor $\mathfrak{\Im}_{12}^{(S)}$ as follows

$$
\mathfrak{\Im}_{11}^{*}=-\mathfrak{\Im}_{12}^{*}=-z \mathfrak{\Im}_{12}^{(S)}, \quad \mathfrak{\Im}_{22}^{*}=-\mathfrak{\Im}_{21}^{*}=-(1-z) \Im_{12}^{(S)}
$$

Thus, all the thermostatic properties of the two-phase material can be expressed with the aid of a single tensor $\Im_{12}^{(S)}$. In that respect, relations (23) and (21) 1 defining phase average $\boldsymbol{\sigma}_{\alpha}^{(\mathrm{in})}$ of internal and external phase stresses reduce to $(\alpha=1,2)$

$$
\begin{aligned}
& \boldsymbol{\sigma}_{1}^{(\mathrm{ex})}=\left[\mathbf{I}+z \mathfrak{\Im}_{12}^{(S)}\left(\boldsymbol{M}_{2}-\boldsymbol{M}_{1}\right)\right] \boldsymbol{\sigma}, \quad \boldsymbol{\sigma}_{1}^{(\mathrm{ex})}=\left[\mathbf{I}-(1-z) \mathfrak{\Im}_{12}^{(S)}\left(\boldsymbol{M}_{2}-\boldsymbol{M}_{1}\right)\right] \boldsymbol{\sigma}, \\
& \boldsymbol{\sigma}_{1}^{(\mathrm{in})}=z \mathfrak{\Im}_{12}^{(S)}\left(\boldsymbol{\Gamma}_{2}-\boldsymbol{\Gamma}_{1}\right), \quad \boldsymbol{\sigma}_{2}^{(\mathrm{in})}=-(1-z) \mathfrak{\Im}_{12}^{(S)}\left(\boldsymbol{\Gamma}_{2}-\boldsymbol{\Gamma}_{1}\right) .
\end{aligned}
$$

The expressions (25) and (26) for effective elastic compliances and macroscopic phase strain become

$$
\begin{aligned}
\boldsymbol{M} & =\boldsymbol{M}^{\mathrm{Re}}-(1-z) z\left(\boldsymbol{M}_{1}-\boldsymbol{M}_{2}\right) \mathfrak{\Im}_{12}^{(S)}\left(\boldsymbol{M}_{1}-\boldsymbol{M}_{2}\right), \quad \boldsymbol{M}^{\mathrm{Re}}=(1-z) \boldsymbol{M}_{1}+z \boldsymbol{M}_{2} \\
\boldsymbol{\varepsilon}^{\mathrm{pt}} & =(1-z) \boldsymbol{\Gamma}_{1}+z \boldsymbol{\Gamma}_{2}-(1-z) z\left(\boldsymbol{M}_{1}-\boldsymbol{M}_{2}\right) \mathfrak{\Im}_{12}^{(S)}\left(\boldsymbol{\Gamma}_{1}-\boldsymbol{\Gamma}_{2}\right)
\end{aligned}
$$

The only nonzero coefficients $g_{\alpha \beta}^{\mathrm{el}}$ and $\phi_{\alpha \beta}^{(0) \text { coh }}$ of the complementary energy and coherency energy cf. (27) and (28), take the form

$$
\begin{gathered}
g_{12}^{\mathrm{el}}=g_{21}^{\mathrm{el}}=\frac{1}{2} \boldsymbol{\sigma} \cdot\left(\boldsymbol{M}_{1}-\boldsymbol{M}_{2}\right) \Im_{12}^{(S)}\left(\boldsymbol{M}_{1}-\boldsymbol{M}_{2}\right) \boldsymbol{\sigma} / \rho, \quad\left(\rho g^{\mathrm{el}}=\frac{1}{2} \boldsymbol{\sigma} \cdot \boldsymbol{M} \boldsymbol{\sigma}\right) \\
\phi_{12}^{(0) \mathrm{coh}}=\phi_{21}^{(0) \mathrm{coh}}=\frac{1}{2}\left(\boldsymbol{\Gamma}_{1}-\boldsymbol{\Gamma}_{2}\right) \cdot \Im_{12}^{(S)}\left(\boldsymbol{\Gamma}_{1}-\boldsymbol{\Gamma}_{2}\right) / \rho, \quad\left(\rho \phi^{\mathrm{coh}}=\Delta \boldsymbol{\Gamma}_{12} \cdot \Im_{12}^{(S)} \Delta \boldsymbol{\Gamma}_{12}\right)
\end{gathered}
$$


Depending on the specific targets of analytical works different approaches may be advantageous in reaching useful estimates.

In practical engineering approach a viable option might be experimental determination of components of effective elastic compliance, which will serve for estimation of other properties. The procedure can take the following path. For $n=2$ we have $\boldsymbol{M}^{\mathrm{Re}}-\boldsymbol{M}_{1}=z_{2}\left(\boldsymbol{M}_{2}-\boldsymbol{M}_{1}\right), \boldsymbol{M}^{\mathrm{Re}}-\boldsymbol{M}_{2}=-z_{1}\left(\boldsymbol{M}_{2}-\boldsymbol{M}_{1}\right)$ and due to that (32) 1 may be rewritten as $\boldsymbol{M}-\boldsymbol{M}^{\mathrm{Re}}=-(1-z) z \Delta \boldsymbol{M}_{12} \Im_{12}^{(S)} \Delta \boldsymbol{M}_{12}$. By solving this equation with respect to $\Im_{12}^{(S)}$ one finds the fundamental relation between $\Im_{12}^{(S)}$ and macroscopic elastic compliance tensor $\boldsymbol{M}$ valid for two-phase microstructure

$$
z_{1} z_{2} \mathfrak{\Im}_{12}^{(S)}=\left(\Delta \boldsymbol{M}_{12}\right)^{-1}\left(\boldsymbol{M}^{\mathrm{Re}}-\boldsymbol{M}\right)\left(\Delta \boldsymbol{M}_{12}\right)^{-1}
$$

Upon experimental determination of effective elastic components their further combination in (34) with (31), $(32)_{2}$ and (33) furnishes expressions for phase averages of all basic thermostatic quantities in terms of the effective elastic compliance $\boldsymbol{M}$.

The formulas presented in this subsection concerning two-phase SMA material were earlier presented by Bernardini [2]. The following correspondence in notation is valid for the tensor $\boldsymbol{R}=-z_{1} z_{2} \Im_{12}^{(S)}$ introduced by formula (62) in [2] and $\mathfrak{\Im}_{12}^{(S)}$. Please note that formula (34) is valid only for macroelement with nonhomogeneous elastic properties $\left(\boldsymbol{M}_{2} \neq \boldsymbol{M}_{1}\right)$. Otherwise the $\Im_{12}^{(S)}=\boldsymbol{L}$ in accordance with its defining formulas (13) $)_{1},(17)$ and (30), $\boldsymbol{L}$ meaning in these cases homogeneous elastic stiffness tensor.

In order to facilitate establishing link between the present developments and classical analytical and/or computational mechanics-oriented studies of heterogeneous media presented for example in $[5,8,14]$ or recently [6] it is worth to specify explicitly relations between moduli $\mathfrak{\Im}_{\alpha \beta}^{*}\left(\Im_{\alpha \beta}^{(S)}, \mathfrak{\Im}_{\alpha \beta}^{(A)}\right.$-cf. (17)) and classical in general diagonally nonsymmetric, dimensionless, fourth-order tensors $\boldsymbol{B}_{\alpha}$ called stress concentration tensors, cf. e.g., Hill [8]—or mechanical concentration factor tensors in [5] $\left(\boldsymbol{\sigma}_{\alpha}^{(\mathrm{ex})}=\boldsymbol{B}_{\alpha} \boldsymbol{\sigma}\right)$. They are as follows

$$
\boldsymbol{B}_{\alpha}=\mathbf{I}+\sum_{\beta=1}^{n} \mathfrak{\Im}_{\alpha \beta}^{*} \boldsymbol{M}_{\beta}, \quad \boldsymbol{B}_{\alpha}=\mathbf{I}+\sum_{\beta=1, \beta \neq \alpha}^{n} z_{\beta}\left[\mathfrak{\Im}_{\alpha \beta}^{(S)} \Delta \boldsymbol{M}_{\beta \alpha}+\mathfrak{\Im}_{\alpha \beta}^{(A)} \boldsymbol{M}_{\beta}\right]
$$

The relations (35) are exact in the sense that they concern the phase averages obtained from the solutions of two different problems of elasticity-first the mechanical problem with $\breve{\boldsymbol{\Gamma}}_{\beta}(\boldsymbol{x})=0$ and uniform surface tractions and second the nonzero eigenstrains problem with vanishing surface tractions. The geometry of microstructure and distribution of moduli of elasticity are in both problems kept the same.

In the case of two-phase materials there exist mathematically exact reciprocal relations between the ingredient $\Im_{12}^{(S)}$ and $\boldsymbol{B}_{\boldsymbol{\alpha}}$. They can be expressed as follows

$$
\mathfrak{\Im}_{12}^{*}=z_{2}\left(\boldsymbol{B}_{1}-\boldsymbol{B}_{2}\right)\left(\boldsymbol{M}_{2}-\boldsymbol{M}_{1}\right)^{-1}, \quad \mathfrak{\Im}_{12}^{(S)}=\left(\boldsymbol{B}_{1}-\boldsymbol{B}_{2}\right)\left(\boldsymbol{M}_{2}-\boldsymbol{M}_{1}\right)^{-1}
$$

When field $\breve{B}(\boldsymbol{x})$ has been determined as a solution of specific boundary-value problem of micromechanics, analytically or with the aid of numerical methods, e.g., finite elements method (FEM), then the values of tensors $\boldsymbol{B}_{\alpha}=\left\langle\breve{B}_{\alpha}(\boldsymbol{x})\right\rangle_{V_{\alpha}}$ can be computed with suitable averaging procedure. Their further combination in (36) $)_{5}$ into (31), (32) 2 and (33) furnishes expressions for phase averages of all basic local quantities and effective thermoelastic properties in terms of stress concentration factor tensors $\boldsymbol{B}_{\boldsymbol{\alpha}}$.

In the case of mixture of more than two phases, in general there do not exist exact inverse relations enabling determination of $\mathfrak{\Im}_{\alpha \beta}^{*}$ in terms of $\boldsymbol{B}_{\alpha}$ as discussed in Dvorak and Benveniste [5]. Therefore, the knowledge of $\boldsymbol{B}_{\alpha}$ is insufficient to evaluate, e.g., the coherency energy of RVE consisting of more than two phases. However, Dvorak and Benveniste [5] give approximate relation estimated by the self-consistent and Mori-Tanaka methods for $\mathfrak{\Im}_{\alpha \beta}^{*}$, that satisfies (16),

$$
\Im_{\alpha \beta}^{*}=\left(I-\boldsymbol{B}_{\alpha}\right)\left(\boldsymbol{M}_{\alpha}-\boldsymbol{M}\right)^{-1}\left(z_{\beta}\left(\boldsymbol{B}_{\beta}\right)^{\mathrm{T}}-\delta_{\alpha \beta} \mathbf{I}\right)
$$

This formula becomes mathematically exact for $n=2$. The $\delta_{\alpha \beta}$ denotes $n$-dimensional Kronecker symbol. It is also of interest to note that phase strain $\boldsymbol{\varepsilon}_{\alpha}^{(\mathrm{in})}$ defined in (26) is not equal to the summand $\boldsymbol{B}_{\alpha}^{T} \boldsymbol{\Gamma}_{\alpha},(! \alpha)$. However, it can be shown that $\sum_{\alpha=1}^{n} z_{\alpha}\left(\boldsymbol{B}_{\alpha}^{\mathrm{T}} \boldsymbol{\Gamma}_{\alpha}-\boldsymbol{\varepsilon}_{\alpha}^{(\mathrm{in})}\right)=0$. Thus, $\boldsymbol{\varepsilon}^{\mathrm{pt}}=\sum_{\alpha=1}^{n} z_{\alpha} \boldsymbol{B}_{\alpha}^{\mathrm{T}} \boldsymbol{\Gamma}_{\alpha}$ demonstrates the possibility of another proportional decomposition of the macroscopic phase strain $\varepsilon^{\mathrm{pt}}$. 


\section{Examples of consistent SEIM estimations of SMA materials thermostatic properties}

\subsection{Reuss-type consistent bounding relationships}

For anisotropic aggregate RVE of $n$ anisotropic phases mixture, the estimate of $\boldsymbol{M}$ by the Reuss upper bound of compliance moduli requires setting $\Im_{\alpha \beta}^{* \operatorname{Re}}=0\left(\Im_{\alpha \beta}^{\operatorname{Re}(S)}=0\right.$ and $\left.\Im_{\alpha \beta}^{\operatorname{Re}(A)}=0\right)$. These subsequently implies $\Re_{\alpha}^{\mathrm{Re}}=0, \mathrm{f}_{\alpha \beta}^{\mathrm{Re}}=0$-cf. (21) what leads to vanishing of $\boldsymbol{M}_{\alpha \beta}^{\mathrm{Re}}=0, g_{\alpha \beta}^{\mathrm{el}}=0$ and the coherency energy $\phi^{\mathrm{coh}}=0$ upon $(25)_{2},(27)_{3}$ and (29), respectively. The following relations are then valid, cf. (23)-(29),

$$
\begin{aligned}
\mathfrak{\Im}_{\alpha \beta}^{* \mathrm{Re}} & =0 \Rightarrow \boldsymbol{M}=\boldsymbol{M}^{\mathrm{Re}}, \boldsymbol{\sigma}_{\alpha}^{(\mathrm{ex})}=\boldsymbol{\sigma}_{\alpha}=\boldsymbol{\sigma}, \boldsymbol{\sigma}_{\alpha}^{(\mathrm{in})}=0, \boldsymbol{\varepsilon}_{\alpha}^{(\mathrm{in})}=\boldsymbol{\Gamma}_{\alpha}, \\
g^{\mathrm{el}} & =\frac{1}{2} \boldsymbol{\sigma} \cdot \boldsymbol{M}^{\mathrm{Re}} \boldsymbol{\sigma}, \quad \phi^{\mathrm{coh}}=0, \quad \boldsymbol{\varepsilon}^{\mathrm{pt}}=\sum_{\alpha=1}^{n} z_{\alpha} \boldsymbol{\Gamma}_{\alpha} . \\
\rho g^{(\mathrm{Re})} & =\sum_{\alpha=1}^{n} z_{\alpha} \rho\left(u_{\alpha}^{0}-T s_{\alpha}^{0}\right)+c_{p}\left[\Delta T-T \ln \left(T / T_{0}\right)\right]-\Delta T \boldsymbol{\sigma} \cdot \boldsymbol{\alpha}-0.5 \boldsymbol{\sigma} \cdot \boldsymbol{M}^{\mathrm{Re}} \boldsymbol{\sigma}-\boldsymbol{\sigma} \cdot \boldsymbol{\varepsilon}^{\mathrm{pt}}
\end{aligned}
$$

The Reuss estimate of Gibbs free energy $g^{(\mathrm{Re})}$ has been obtained upon substitution of respective earlier terms and properties of (38) into (1) . Naturally the maximum value of number of phases must be replaced with $n$.

The properties (38) would also result from the rigorous solutions of UT-uniform traction, boundary-value problems for a class of specific microstrain fields $\boldsymbol{M}_{\alpha} \boldsymbol{\sigma}$ and $\boldsymbol{\Gamma}_{\alpha} \quad\left(\boldsymbol{x} \in V_{\alpha}, \alpha=1 \ldots n\right)$, compatible at all interfaces and for special microstructural geometries, the ones leading to uniform stresses all over RVE. When compatibility conditions are not satisfied the results (38) following from the assumption $\Im_{\alpha \beta}^{* \mathrm{Re}}=0$ can be treated as special estimates, constituting some type of bound for the RVE effective properties at UT boundary conditions. For example $\boldsymbol{M}^{R e}$ is an upper bound to the UT effective elastic compliance in the sense that the corresponding macroscopic elastic energy $0.5 \sigma \cdot M^{\mathrm{Re}} \boldsymbol{\sigma}$ is not lesser than the actual macroscopic energy-cf., e.g., Nemat-Nasser and Hori [14], and $\phi^{\text {coh }}=0$ is the lower bound to the coherency energy.

\subsection{Voigt-type consistent bounding relationships}

The derivation of the specific form of stress concentration ingredients describing Voigt-type estimations of various multiphase material properties is slightly more involved. Let us consider the following two mathematically equivalent relations between Reuss (upper bound of effective compliance $\boldsymbol{M}$ ) and Voigt (upper bound of effective stiffness $\boldsymbol{L}=\boldsymbol{M}^{-1}$ ) moduli,

$$
\begin{aligned}
& \boldsymbol{M}^{\mathrm{Re}}-\boldsymbol{L}^{V}=\sum_{\alpha, \beta=1,}^{n} z_{\alpha \neq \alpha} z_{\beta} \boldsymbol{M}_{\alpha \beta}^{V}, \quad \boldsymbol{L}^{V} \equiv \sum_{\alpha=1}^{n} z_{\alpha} \boldsymbol{L}_{\alpha}, \quad\left(\boldsymbol{L}_{\alpha}=\boldsymbol{M}_{\alpha}^{-1}\right), \\
& \boldsymbol{M}_{\alpha \beta}^{V} \equiv\left(-\frac{1}{4}\right)\left(\boldsymbol{L}^{V} \Delta \boldsymbol{L}_{\alpha \beta} \Delta \boldsymbol{M}_{\alpha \beta}+\Delta \boldsymbol{M}_{\alpha \beta} \Delta \boldsymbol{L}_{\alpha \beta} \boldsymbol{L}^{V}\right), \\
& \boldsymbol{M}_{\alpha \beta}^{V}=0.5\left(\Delta \boldsymbol{M}_{\alpha \beta} \boldsymbol{L}_{\alpha \beta}^{(\mathrm{S})} \Delta \boldsymbol{M}_{\alpha \beta}-\boldsymbol{M}_{\alpha} \boldsymbol{L}_{\alpha \beta}^{(\mathrm{A})} \boldsymbol{M}_{\beta}+\boldsymbol{M}_{\beta} \boldsymbol{L}_{\alpha \beta}^{(\mathrm{A})} \boldsymbol{M}_{\alpha}\right), \quad(! \alpha, \beta)
\end{aligned}
$$

where

$$
\begin{aligned}
& \boldsymbol{L}_{\alpha \beta} \equiv \boldsymbol{L}_{\alpha} \boldsymbol{L}^{V} \boldsymbol{L}_{\beta}, \quad \boldsymbol{L}_{\alpha}=z_{\alpha} \boldsymbol{L}_{\alpha \alpha}^{(\mathrm{S})}+\sum_{\alpha, \beta=1 ; \alpha \neq \beta}^{n} z_{\beta} \boldsymbol{L}_{\alpha \beta}^{(\mathrm{S})}, \quad \Delta \boldsymbol{L}_{\alpha \beta} \equiv \boldsymbol{L}_{\alpha}-\boldsymbol{L}_{\beta}, \quad \Delta \boldsymbol{M}_{\alpha \beta} \equiv \boldsymbol{M}_{\alpha}-\boldsymbol{M}_{\beta}, \\
& \boldsymbol{L}_{\alpha \beta}^{(\mathrm{S})}=\frac{1}{2}\left(\boldsymbol{L}_{\alpha \beta}+\boldsymbol{L}_{\beta \alpha}\right), \quad \boldsymbol{L}_{\alpha \beta}^{(\mathrm{A})}=\frac{1}{2}\left(\boldsymbol{L}_{\alpha \beta}-\boldsymbol{L}_{\beta \alpha}\right), \quad \boldsymbol{L}_{\alpha \beta}^{(S)}=\boldsymbol{L}_{\alpha \beta}^{(S)}, \quad \boldsymbol{L}_{\alpha \beta}^{\mathrm{T}}=-\boldsymbol{L}_{\alpha \beta}^{(A)}
\end{aligned}
$$

The expressions (39)-(40) correspond to relations (25) in the situation when for specific aggregate special microgeometry exists (leading to uniform strains all over RVE) resulting in effective elastic compliance equal to $\boldsymbol{M}=\left(\boldsymbol{L}^{V}\right)^{-1}$.

The physical equivalence concerns the points of the constraint plane $\Sigma_{\alpha=1}^{n} z_{\alpha}=1$ and should be understood in a proper context: for fixed $T, \boldsymbol{\sigma}, \boldsymbol{\Gamma}_{\alpha}$ and $\boldsymbol{h}$ there are many functions of $z_{\alpha},(\alpha=1, \ldots, n)$ describing a 
physical property. Two different functions that reach the same value in each point of the plane $\Sigma_{\alpha=1}^{n} z_{\alpha}=1$ are equivalent. To obtain (39) 1 and equivalence (40) one uses the identities

$$
\sum_{\alpha=1}^{n} z_{\alpha}^{2}=1-\sum_{\alpha, \beta=1, \alpha \neq \beta}^{n} z_{\alpha} z_{\beta}, \boldsymbol{M}_{\alpha} \boldsymbol{L}_{\beta}+\boldsymbol{M}_{\beta} \boldsymbol{L}_{\alpha}=2 \mathbf{I}-\Delta \boldsymbol{M}_{\alpha \beta} \Delta \boldsymbol{L}_{\alpha \beta}
$$

By comparing $(25)_{2}$ with $(40)_{2}$ it is deduced that the ingredients of stress concentration moduli associated with the Voigt bounds $\Im_{\alpha \beta}^{* V}-$ cf. (17), are $(\alpha \neq \beta)$,

$$
\begin{aligned}
& \Im_{\alpha \beta}^{V(S)}=\boldsymbol{L}_{\alpha \beta}^{(S)}, \quad \Im_{\alpha \beta}^{V(A)}=\boldsymbol{L}_{\alpha \beta}^{(A)} \quad \text { or equivalently } \\
& \mathfrak{\Im}_{\alpha \beta}^{V(S)}=\left(\Delta \boldsymbol{M}_{\alpha \beta}\right)^{-1}\left(2 \boldsymbol{M}_{\alpha \beta}^{V}\right)\left(\Delta \boldsymbol{M}_{\alpha \beta}\right)^{-1} \quad(! \alpha, \beta), \quad \Im_{\alpha \beta}^{V(A)}=0
\end{aligned}
$$

The ingredients satisfy condition (19) 2 and tend to $\boldsymbol{L}_{\alpha}$ when $z_{\beta} \rightarrow$ 1, i.e., $\left.\mathfrak{\Im}_{\alpha \beta}^{V(S)}\right|_{z_{\beta} \rightarrow 1}=\boldsymbol{L}_{\alpha}$.

Consequently this estimation predicts that the phase average $\sigma_{\alpha}^{(\text {in) }}$ of internal stress in the product phase $\alpha$ at the beginning of $\beta \rightarrow \alpha$ p.t. equals to the stress that could occur in an inclusion embedded into a rigid matrix, i.e., $\left.\boldsymbol{\sigma}_{\alpha}^{(\mathrm{in})}\right|_{z_{\beta}=1}=-\boldsymbol{L}_{\alpha} \boldsymbol{\Gamma}_{\alpha}$, provided that $\boldsymbol{\Gamma}_{\beta}=0$. It is also worthwhile to note that in the case of two-phase mixture $(n=2) \quad \Im_{\alpha \beta}^{V(\mathrm{~A})}=\boldsymbol{L}_{\alpha \beta}^{(\mathrm{A})}=0$, identically.

Consistent with the reciprocity theorem Voigt-type estimation of all thermostatic properties defined on the plane $\Sigma_{\alpha=1}^{n} z_{\alpha}=1$ may be found by substituting (43) into formulas presented in Sect. 4.2. Using (43) 1,2 and the connections for $\boldsymbol{\Gamma}_{\gamma}-\mathrm{cf}$. (22),

$$
\begin{aligned}
\mathfrak{R}_{\alpha}^{V}\left(z_{\gamma}, \boldsymbol{\Gamma}_{\gamma}\right) & =\sum_{\beta=1, \beta \neq \alpha}^{n} z_{\beta}\left[\boldsymbol{L}_{\alpha \beta}^{(\mathrm{S})}\left(\boldsymbol{\Gamma}_{\beta}-\boldsymbol{\Gamma}_{\alpha}\right)+\boldsymbol{L}_{\alpha \beta}^{(\mathrm{A})} \boldsymbol{\Gamma}_{\beta}\right] \Rightarrow \mathfrak{R}_{\alpha}^{V}\left(z_{\gamma}, \boldsymbol{\Gamma}_{\gamma}\right)=\boldsymbol{L}_{\alpha}\left[\boldsymbol{\varepsilon}_{0}^{P}-\boldsymbol{\Gamma}_{\alpha}\right] \\
\boldsymbol{\varepsilon}_{0}^{P}\left(z_{\gamma}, \boldsymbol{\Gamma}_{\gamma}\right) & \equiv \sum_{\beta=1}^{n} z_{\beta} \boldsymbol{L}^{V} \boldsymbol{L}_{\beta} \boldsymbol{\Gamma}_{\beta} .
\end{aligned}
$$

Collected together Voigt-type estimations resulting from (43) corresponding to the Reuss-type estimations (38), displaying uniform distribution of strain, are

$$
\begin{aligned}
& \boldsymbol{M}=\stackrel{-}{V}^{V}, \boldsymbol{\sigma}_{\alpha}^{(\mathrm{ex})}=\boldsymbol{L}_{\alpha} \boldsymbol{\varepsilon}_{\alpha}^{(\mathrm{ex})}, \boldsymbol{\varepsilon}_{\alpha}^{(\mathrm{ex})}={ }^{-1} \boldsymbol{L}^{V} \boldsymbol{\sigma}, \boldsymbol{\sigma}_{\alpha}^{(\mathrm{in})}\left(z_{\gamma}, \boldsymbol{\Gamma}_{\gamma}\right)=\boldsymbol{L}_{\alpha}\left[\boldsymbol{\varepsilon}_{0}^{P}-\boldsymbol{\Gamma}_{\alpha}\right], \boldsymbol{\varepsilon}_{\alpha}^{(\mathrm{in})}=\boldsymbol{\varepsilon}_{0}^{P}, \boldsymbol{\varepsilon}^{\mathrm{pt}}=\boldsymbol{\varepsilon}_{0}^{P}, \\
& \rho g^{\mathrm{el}}\left(z_{\gamma}, \boldsymbol{\sigma}\right)=0.5 \boldsymbol{\sigma} \cdot \boldsymbol{L}^{V} \boldsymbol{\sigma}, \quad \rho \phi^{\mathrm{coh}}\left(z_{\gamma}, \boldsymbol{\Gamma}_{\gamma}\right)=0.5 \sum_{\alpha=1}^{n} z_{\alpha}\left(\boldsymbol{\Gamma}_{\alpha}-\boldsymbol{\varepsilon}_{0}^{P}\right) \cdot \boldsymbol{L}_{\alpha}\left(\boldsymbol{\Gamma}_{\alpha}-\boldsymbol{\varepsilon}_{0}^{P}\right) \geq 0 .
\end{aligned}
$$

The Voigt-type estimation of Gibbs free energy $g^{(V)}$ takes the form

$$
\begin{aligned}
\rho g^{(V)} & =\left(\sum_{\alpha=1}^{n} z_{\alpha} \rho g_{\alpha}^{0}\right)-0.5 \boldsymbol{\sigma} \cdot \boldsymbol{L}^{V} \boldsymbol{\sigma}-\boldsymbol{\sigma} \cdot \boldsymbol{\varepsilon}_{0}^{P}+0.5 \sum_{\alpha=1}^{n} z_{\alpha}\left(\boldsymbol{\Gamma}_{\alpha}-\boldsymbol{\varepsilon}_{0}^{P}\right) \cdot \boldsymbol{L}_{\alpha}\left(\boldsymbol{\Gamma}_{\alpha}-\boldsymbol{\varepsilon}_{0}^{P}\right), \\
g_{\alpha}^{0} & =u_{\alpha}^{0}-T s_{\alpha}^{0}+c_{p}\left[\Delta T-T \ln \left(T / T_{0}\right)\right]-\Delta T \boldsymbol{\sigma} \cdot \boldsymbol{\alpha} / \rho .
\end{aligned}
$$

The total strains of all phases are equal to $\varepsilon_{\alpha}=\left(\boldsymbol{L}^{V}\right)^{-1} \boldsymbol{\sigma}+\boldsymbol{\varepsilon}_{0}^{P}+\boldsymbol{\alpha} \Delta T$. The Voigt-type estimation of the macroscopic coherency energy (29) can be alternatively presented in the form $\rho \phi_{\alpha \beta}^{(0) \text { coh }}=0.5\left(\boldsymbol{\Gamma}_{\alpha} \cdot \boldsymbol{L}_{\alpha \beta}^{(\mathrm{S})} \boldsymbol{\Gamma}_{\alpha}+\right.$ $\left.\boldsymbol{\Gamma}_{\beta} \cdot \boldsymbol{L}_{\alpha \beta}^{(\mathrm{S})} \boldsymbol{\Gamma}_{\beta}\right)-\boldsymbol{\Gamma}_{\alpha} \cdot \boldsymbol{L}_{\alpha \beta} \boldsymbol{\Gamma}_{\beta}$. The partial phase ultimate phase eigenstrains can be expressed as follows

$$
\boldsymbol{\kappa}_{\alpha}\left(z_{\gamma}, \boldsymbol{\Gamma}_{\gamma}\right)=\overline{\boldsymbol{L}}^{V} \boldsymbol{L}_{\alpha} \boldsymbol{\Gamma}_{\alpha}+\left(I-\bar{L}^{V} \boldsymbol{L}_{\alpha}\right) \boldsymbol{\varepsilon}_{0}^{P}\left(z_{\gamma}, \boldsymbol{\Gamma}_{\gamma}\right), \quad\left(\boldsymbol{\varepsilon}^{\mathrm{pt}}=\sum_{\alpha=1}^{n} z_{\alpha} \boldsymbol{\kappa}_{\alpha}\right) .
$$




\section{Special class of experiment-oriented SEIM estimation $\left(A_{\alpha \beta}\right)$}

Finding the manner for introducing into explicit mathematical formulas adequate parametric objects in such a way that it is possible in modeling description, in convenient and efficient way, taking into account influence of inhomogeneous elastic properties, inhomogeneous or incompatible eigenstrains, and/or evolution of microstructure on effective thermoelastic properties of macroelement still poses an open scientific problem. Elucidating clue for such an operation is delivered by two equivalent forms of formulas (43) 1,2 and $(43)_{3,4}$ for tensors $\Im_{\alpha \beta}^{V(\mathrm{~S})}$. Let us accept conjecture that for $n$ nonisotropic phases generating nonisotropic aggregate RVE the ingredients of stress concentration moduli $\Im_{\alpha \beta}^{*}-\mathrm{cf}$. (20), have the following form $(\alpha \neq \beta)(! \alpha)$,

$$
\begin{aligned}
\mathfrak{\Im}_{\alpha \beta}^{V+A_{\alpha \beta}(S)} & =-0.5\left[\Delta \boldsymbol{L}_{\alpha \beta} \boldsymbol{L}_{\alpha \beta}^{V+A_{\alpha \beta}} \Delta \boldsymbol{M}_{\alpha \beta}^{-1}+\Delta \boldsymbol{M}_{\alpha \beta}^{-1} \boldsymbol{L}_{\alpha \beta}^{-1} \operatorname{An}_{\alpha \beta}^{-1} \Delta \boldsymbol{L}_{\alpha \beta}\right], \quad \Im_{\alpha \beta}^{V+A_{\alpha \beta}(A)}=0, \\
\boldsymbol{L}_{\alpha \beta}^{V+A_{\alpha \beta}} & \equiv \boldsymbol{L}^{V}+\boldsymbol{A}_{\alpha \beta}, \quad \boldsymbol{A}_{\alpha \alpha}=0(! \alpha), \boldsymbol{A}_{\alpha \beta}=\boldsymbol{A}_{\beta \alpha}, \boldsymbol{A}_{\alpha \beta}^{\mathrm{T}}=\boldsymbol{A}_{\alpha \beta}, \\
\mathfrak{\Im}_{\alpha \beta}^{V+A_{\alpha \beta}(S)} & =\Delta \boldsymbol{M}_{\alpha \beta}^{-1} 2 \boldsymbol{M}_{\alpha \beta}^{V+A_{\alpha \beta}} \Delta \boldsymbol{M}_{\alpha \beta}^{-1}, \quad \boldsymbol{M}_{\alpha \beta}^{V+A_{\alpha \beta}} \\
& =\left(-\frac{1}{4}\right)\left[\Delta \boldsymbol{M}_{\alpha \beta} \Delta \boldsymbol{L}_{\alpha \beta} \boldsymbol{L}_{\alpha \beta}^{V+A_{\alpha \beta}}+\boldsymbol{L}_{\alpha \beta}^{V+A_{\alpha \beta}} \Delta \boldsymbol{L}_{\alpha \beta} \Delta \boldsymbol{M}_{\alpha \beta}\right]
\end{aligned}
$$

The symbol $\boldsymbol{L}^{V}$ denotes Voigt upper bound of the elastic stiffness moduli-cf. (39)2, whereas $\Delta \boldsymbol{M}_{\alpha \beta}$ and $\Delta \boldsymbol{L}_{\alpha \beta}$ are defined in (41). Tensors $\boldsymbol{A}_{\alpha \beta}$ make an array of $n(n-1) / 2$ independent positive semidefinite, diagonally symmetric fourth-order tensors. The tensors $\boldsymbol{A}_{\alpha \beta}$ may, in general, depend on phase composition and parameters describing the current geometry of RVE microstructure. Thus, family of such tensors can conveniently describe not only impact of nonhomogeneous elastic properties of specific phases-usually assumed to be invariable in time, on effective thermoelastic properties of SMA macroelement but also influences resulting from changing due to active phase transitions microstructure.

The estimations based on (48) have an interesting feature that all effective properties become the Voigttype and Reuss-type estimations when $\boldsymbol{A}_{\alpha \beta}=0$ and $\boldsymbol{A}_{\alpha \beta} \rightarrow \infty$, respectively, provided that some of $\boldsymbol{M}_{\alpha}$ are different. Relations (48) make a very convenient tool for obtaining precise assessments of thermomechanical properties for various microstructures but usually this will require rather involved numerical-experimental studies to reach useful results. For example Patoor et. al [17] in efforts to develop microscopically motivated macroscopic SMA model as a basic ingredients distinguished habit plane variants of martensite $(n=24$ for $\mathrm{CuZnAl}$ alloy) and introduced so-called interaction matrix $H^{m n}$ characterizing energy interactions between the martensitic variants. Some modeling results and discussion on advantages and drawbacks of this approach can be found in, e.g., Peultier et. al. [18] and/or Lagoudas et. al. [9]. Developed here approach upon its use in well-designed numerical tests can deliver much deeper understanding on the structure and influence of interaction matrix on the coherency energy. The very comprehensive route taking advantage of the matrix of tensors $\boldsymbol{A}_{\alpha \beta}$ very well suited for fundamental research will be now put aside for future studies. Instead further simplifications will be adopted of the three kinds: a) the matrix of tensors $\boldsymbol{A}_{\alpha \beta}$ will be reduced to one tensor $\boldsymbol{A}_{\alpha \beta}=\boldsymbol{A}, \mathrm{b}$ ) attention will be focused on isotropic aggregates, and c) number of distinguished phases will be limited.

\subsection{Two parameter estimation for isotropic aggregate of $n$ isotropic phases $\left(\boldsymbol{A}_{\alpha \beta}=\boldsymbol{A}\right)$}

Obtaining useful estimations with limited/reasonable computational and/or experimental tests requires simplifications. In particular interesting course is the one involving only one tensor $\boldsymbol{A}$, i.e., conjecture that it can be accepted that all $\boldsymbol{A}_{\alpha \beta}$ coincide $\left(\boldsymbol{A}_{\alpha \beta}=\boldsymbol{A}\right)$. The tensor $\boldsymbol{A}$ is a positive semidefinite diagonally symmetric fourthorder tensor regarded here as empirical quantity, which is to be determined in a course of an appropriately designed experimental program. In general, it may depend on phase composition and parameters describing the current geometry of microstructure. In that case all $\boldsymbol{L}_{\alpha \beta}^{V+A}$ defined in (48) $)_{3}$ reduce to a single tensor $\boldsymbol{L}^{V+A} \equiv \boldsymbol{L}^{V}+\boldsymbol{A}=\boldsymbol{L}_{\alpha \beta}^{V+A_{\alpha \beta}}$. The attraction of such an approach relies on the fact that upon this conjecture and assumptions (48) $)_{1,2}$ all the effective properties may be consistently expressed in terms of estimation of 
elastic compliance $\boldsymbol{M}$, in particular with the aid of its experimentally determined components. To show this let us proceed as follows.

From (39) $)_{1},(40)_{1}$ and definition of $\boldsymbol{L}^{V+A}$ it can be shown that $\boldsymbol{M}-\left(\boldsymbol{L}^{V+A}\right)^{-1}=\boldsymbol{M}^{\mathrm{Re}} \boldsymbol{A}\left(\boldsymbol{L}^{V+A}\right)^{-1}$, upon taking advantage of the property $\left(\boldsymbol{L}^{V+A}\right)^{-1} \Delta \boldsymbol{L}_{\alpha \beta} \Delta \boldsymbol{M}_{\alpha \beta}=\Delta \boldsymbol{M}_{\alpha \beta} \Delta \boldsymbol{L}_{\alpha \beta}\left(\boldsymbol{L}^{V+A}\right)^{-1}$ resulting from diagonal symmetry of all tensors present in this last relation. Next upon using identity $\boldsymbol{A}\left(\boldsymbol{L}^{V+A}\right)^{-1}=$ $\boldsymbol{I}-\boldsymbol{L}^{V}\left(\boldsymbol{L}^{V+A}\right)^{-1}$-resulting directly from definition of $\boldsymbol{L}^{V+A}$, and simple manipulations one obtains

$$
\begin{gathered}
\boldsymbol{M}^{\mathrm{Re}}-\boldsymbol{M}=0.5\left[\left(\boldsymbol{M}^{\mathrm{Re}} \boldsymbol{L}^{V}-\boldsymbol{I}\right) \boldsymbol{L}^{-1}+\boldsymbol{L}^{-1}\left(\boldsymbol{L}^{V} \boldsymbol{M}^{\mathrm{Re}}-\boldsymbol{I}\right)\right], \quad \boldsymbol{M}^{\mathrm{Re}}-\boldsymbol{M}=\sum_{\alpha, \beta=1, \beta \neq \alpha}^{n} z_{\alpha} z_{\beta} \boldsymbol{M}_{\alpha \beta}^{V+A}, \\
\boldsymbol{L}^{V+A} \equiv \boldsymbol{L}^{V}+\boldsymbol{A}=\boldsymbol{L}_{\alpha \beta}^{V+\boldsymbol{A}_{\alpha \beta}}, \quad \boldsymbol{A}^{\mathrm{T}}=\boldsymbol{A}, \quad \boldsymbol{M}_{\alpha \beta}^{V+A}=\left(-\frac{1}{4}\right)\left[\boldsymbol{L}^{-1} \Delta+A \boldsymbol{L}_{\alpha \beta} \Delta \boldsymbol{M}_{\alpha \beta}+\Delta \boldsymbol{M}_{\alpha \beta} \Delta \boldsymbol{L}_{\alpha \beta} \boldsymbol{L}^{-1}\right] .
\end{gathered}
$$

Expression (49) 4 for $\boldsymbol{M}_{\alpha \beta}^{V+A}$ is reached upon inserting (48)2,3,4 with $\boldsymbol{L}_{\alpha \beta}^{V+\boldsymbol{A}_{\alpha \beta}}=\boldsymbol{L}^{V+A}$ into (25) . The connection (49) 1 may be regarded as the algebraic equation enabling to find unknown components of tensor $\left(\boldsymbol{L}^{V+A}\right)^{-1}$ in terms of $\boldsymbol{M}$ and $\boldsymbol{M}_{\alpha}$. Upon substitution of $\left(\boldsymbol{L}^{V+A}\right)^{-1}$ determined from (49) $)_{1}$ into (48) 1 leads to the eventual expression of all nonzero ingredients of stress concentration $\Im_{\alpha \beta}^{V+A(S)}$ in terms of treated as known $\boldsymbol{M}, \boldsymbol{M}^{\mathrm{Re}}$ and $\boldsymbol{L}^{\boldsymbol{V}}$. Such determined $\Im_{\alpha \beta}^{V+A(S)}$ entered into (48) and used in connections (22), (21) give the following links

$$
\begin{aligned}
\mathfrak{\Im}_{\alpha \beta}^{V+A(S)} & =\left(\Delta \boldsymbol{M}_{\alpha \beta}\right)^{-1}\left(2 \boldsymbol{M}_{\alpha \beta}^{V+A}\right)\left(\Delta \boldsymbol{M}_{\alpha \beta}\right)^{-1}, \quad \Im_{\alpha \beta}^{(A)}=0, \\
\mathfrak{R}_{\alpha}^{V+A}\left(\boldsymbol{\Gamma}_{\gamma}\right) & =\sum_{\beta=1, \beta \neq \alpha}^{n} z_{\beta}\left(\Delta \boldsymbol{M}_{\alpha \beta}\right)^{-1}\left(2 \boldsymbol{M}_{\alpha \beta}^{V+A}\right)\left(\Delta \boldsymbol{M}_{\alpha \beta}\right)^{-1}\left(\boldsymbol{\Gamma}_{\beta}-\boldsymbol{\Gamma}_{\alpha}\right) \\
\mathbf{f}_{\alpha \beta}^{V+A}(\boldsymbol{X}, \boldsymbol{Y}) & =(\boldsymbol{X}-\boldsymbol{Y}) \cdot\left(\Delta \boldsymbol{M}_{\alpha \beta}\right)^{-1} \boldsymbol{M}_{\alpha \beta}^{V+A}\left(\Delta \boldsymbol{M}_{\alpha \beta}\right)^{-1}(\boldsymbol{X}-\boldsymbol{Y}) \quad(! \alpha, \beta=1,2 \ldots n)
\end{aligned}
$$

The estimation of all thermostatic properties consistent with the reciprocity theorem requires substitution of (50) into relations (23)-(27), (29), (1) to obtain explicit expressions for $\boldsymbol{\sigma}_{\alpha}^{(\mathrm{ex})}, \boldsymbol{\sigma}_{\alpha}^{(\mathrm{in})}, \boldsymbol{\varepsilon}_{\alpha}^{(\mathrm{ex})}, \boldsymbol{\varepsilon}_{\alpha}^{(\mathrm{in})}, \boldsymbol{M}, \boldsymbol{\varepsilon}^{\mathrm{pt}}, g^{\mathrm{el}}, \phi^{\mathrm{coh}}, g$, respectively. They take the same explicit form as the formulas (45)-(47) valid for Voigt-type estimates but in place of $\boldsymbol{L}^{V}$ there must be substituted $\boldsymbol{L}^{V+A}\left(\boldsymbol{L}^{V} \rightarrow \boldsymbol{L}^{V+A}\right)$.

Further considerable simplifications are possible when it can be conjectured that modeled material RVE consisting of $n$ isotropic phases always arranges into isotropic resulting aggregate. In such a case it is convenient to employ the Rychlewski's theorem on spectral decomposition of fourth-order elastic stiffness tensor, see Rychlewski [25] for its accessible exposition. The theorem states that in the case of isotropic linear elastic materials $\boldsymbol{L}(\boldsymbol{M})$ can be uniquely decomposed into spherical and distortional parts, as follows

$$
\begin{gathered}
\boldsymbol{L}_{\alpha}=3 \mathbf{k}_{\alpha} \mathbf{J}+2 \mu_{\alpha} \mathbf{K}, \quad \boldsymbol{M}_{\alpha}=\left(1 / 3 \mathbf{k}_{\alpha}\right) \mathbf{J}+\left(1 / 2 \mu_{\alpha}\right) \mathbf{K}, \quad \mathbf{K}=\mathbf{I}-\mathbf{J}, \quad \mathbf{J}=\frac{1}{3} \boldsymbol{I} \otimes \boldsymbol{1}, \\
\mathbf{J}^{2}=\mathbf{J}, \quad \mathbf{K}^{2}=\mathbf{K}, \quad \mathbf{K} \mathbf{J}=0, \quad \boldsymbol{L}^{V}=3 \mathbf{k}^{V} \mathbf{J}+2 \mu^{V} \mathbf{K}, \quad \boldsymbol{L}^{V}=\frac{1}{3 \mathbf{k}^{V}} \mathbf{J}+\frac{1}{2 \mu^{V}} \mathbf{K}, \\
\boldsymbol{L}^{V+A}=3\left(\mathbf{k}^{V}+A^{(k)}\right) \mathbf{J}+2\left(\mu^{V}+A^{(\mu)}\right) \mathbf{K}, \quad \boldsymbol{A}=3 A^{(k)} \mathbf{J}+2 A^{(\mu)} \mathbf{K}, \quad\left(J_{i j k l}=\frac{1}{3} \delta_{i j} \delta_{k l}\right) .
\end{gathered}
$$

Walpole [29] seems to be the first to use this type of decomposition/notation, and it is also employed in [2]. Then, $\Im_{\alpha \beta}^{V+A^{(S)}}$ present in (50) reduces to the form $(\alpha \neq \beta)$

$$
\begin{aligned}
\mathfrak{\Im}_{\alpha \beta}^{V+A(S)} & =\frac{3 \mathrm{k}_{\alpha} \mathrm{k}_{\beta}}{\mathrm{k}^{V}+A^{(k)}} \mathbf{J}+\frac{2 \mu_{\alpha} \mu_{\beta}}{\mu^{V}+A^{(\mu)}} \mathbf{K}, \quad \Im_{\alpha \beta}^{(A)}=0 \\
\mathrm{k}_{\alpha} & =\frac{E_{\alpha}}{3\left(1-2 v_{\alpha}\right)}, \quad \mu_{\alpha}=\frac{E_{\alpha}}{2\left(1+v_{\alpha}\right)}, \quad \mathbf{k}^{V}=\sum_{\alpha=1}^{n} z_{\alpha} \mathrm{k}_{\alpha}, \quad \mu^{V}=\sum_{\alpha=1}^{n} z_{\alpha} \mu_{\alpha}
\end{aligned}
$$


Here, the symbols $\mathrm{k}^{V}$ and $\mu^{V}$ denote the average value of the bulk moduli $\mathbf{k}_{\alpha}$ and shear moduli $\mu_{\alpha}$ in total volume of RVE. They can be identified, of course, with the Voigt upper bounds of corresponding bulk and shear macroscopic moduli. The symbols $E_{\alpha}$ and $v_{\alpha}$ denote Young's moduli and Poisson's ratios of the phases, respectively.

By substitution of (52) into (25), (27) one finds the following eventual expressions for estimates of effective compliance tensor $\boldsymbol{M}$ (constants k and $\mu$ ),

$$
\begin{gathered}
\boldsymbol{M}=(1 / 3 \mathrm{k}) \mathbf{J}+(1 / 2 \mu) \mathbf{K}, \quad \mathrm{k}=\mathrm{k}^{\mathrm{Re}} \frac{\mathrm{k}^{V}+A^{(\mathrm{k})}}{\mathrm{k}^{\operatorname{Re}}+A^{(\mathrm{k})}}, \quad \mu=\mu^{\operatorname{Re}} \frac{\mu^{V}+A^{(\mu)}}{\mu^{\operatorname{Re}}+A^{(\mu)}} \\
\boldsymbol{M}^{\operatorname{Re}}=\left(1 / 3 \mathrm{k}^{\operatorname{Re}}\right) J+\left(1 / 2 \mu^{\mathrm{Re}}\right) \mathbf{K}, \quad 1 / \mathbf{k}^{\operatorname{Re}}=\sum_{\alpha=1}^{n}\left(z_{\alpha} / \mathbf{k}_{\alpha}\right), \quad 1 / \mu^{\operatorname{Re}}=\sum_{\alpha=1}^{n}\left(z_{\alpha} / \mu_{\alpha}\right)
\end{gathered}
$$

and elastic energy $\rho g^{\mathrm{el}}$,

$$
\begin{aligned}
\rho g^{\mathrm{el}} & =0.5 \boldsymbol{\sigma} \cdot \boldsymbol{M}^{\mathrm{Re}} \boldsymbol{\sigma}-0.5 \sum_{\alpha, \beta=1, \alpha \neq \beta}^{n} z_{\alpha} z_{\beta} \mathrm{f}_{\alpha \beta}\left(\boldsymbol{M}_{\alpha} \boldsymbol{\sigma}, \boldsymbol{M}_{\beta} \boldsymbol{\sigma}\right), \quad \boldsymbol{\sigma} \cdot \boldsymbol{M}^{\mathrm{Re}} \boldsymbol{\sigma}=\frac{p^{2}}{\mathrm{k}^{\mathrm{Re}}}+\frac{\boldsymbol{\sigma}^{\prime} \cdot \boldsymbol{\sigma}^{\prime}}{2 \mu^{\mathrm{Re}}}, \\
f_{\alpha \beta}\left(\boldsymbol{M}_{\alpha} \boldsymbol{\sigma}, \boldsymbol{M}_{\beta} \boldsymbol{\sigma}\right) & =\frac{\left(\mathrm{k}_{\alpha}-\mathrm{k}_{\beta}\right)^{2} p^{2}}{2 \mathrm{k}_{\alpha} \mathrm{k}_{\beta}\left[\mathrm{k}^{V}+A^{(\mathrm{k})}\right]}+\frac{\left(\mu_{\alpha}-\mu_{\beta}\right)^{2} \boldsymbol{\sigma}^{\prime} \cdot \boldsymbol{\sigma}^{\prime}}{4 \mu_{\alpha} \mu_{\beta}\left[\mu^{V}+A^{(\mu)}\right]} \\
p \boldsymbol{l} & =\frac{1}{3}(\boldsymbol{I} \otimes \boldsymbol{l}) \boldsymbol{\sigma}=\mathbf{J} \boldsymbol{\sigma}, \quad \boldsymbol{\sigma}^{\prime}=\operatorname{dev} \boldsymbol{\sigma}=\boldsymbol{\sigma}-\mathbf{J} \boldsymbol{\sigma}=\mathbf{K} \boldsymbol{\sigma}, \quad p=\frac{1}{3} \operatorname{tr} \boldsymbol{\sigma}, \quad \operatorname{dev} \boldsymbol{\sigma} \equiv \boldsymbol{\sigma}-\frac{1}{3} \operatorname{tr} \boldsymbol{}
\end{aligned}
$$

The superscript "Re" denotes Reuss lower bounds of macroscopic, isotropic shear and bulk elastic moduli $1 / \mu^{\mathrm{Re}}$ and $1 / k^{\mathrm{Re}}$. The following identity valid for arbitrary array $r_{\alpha}$ was used to get (53)

$$
\frac{r^{V}}{r^{\operatorname{Re}}}=1+\frac{1}{2} \sum_{\alpha, \beta=1, \alpha \neq \beta}^{n} z_{\alpha} z_{\beta} \frac{\left(r_{\alpha}-r_{\beta}\right)^{2}}{r_{\alpha} r_{\beta}} .
$$

The estimates of effective properties satisfy familiar inequalities—cf. Nemat-Nasser and Hori [14],

$$
\mathrm{k}^{V} \geq \mathrm{k} \geq \mathrm{k}^{\mathrm{Re}}, \quad \mu^{V} \geq \mu \geq \mu^{\mathrm{Re}}, \quad A^{(\mathrm{k})} \geq 0, \quad A^{(\mu)} \geq 0 .
$$

The careful inspection of expression $\boldsymbol{\sigma} \cdot\left(\boldsymbol{M}_{\alpha}-\boldsymbol{M}_{\beta}\right)\left(\boldsymbol{M}_{\alpha}-\boldsymbol{M}_{\beta}\right) \boldsymbol{\sigma}$ written for isotropic tensors shows that to get $f_{\alpha \beta}\left(\boldsymbol{\Gamma}_{\alpha}, \boldsymbol{\Gamma}_{\beta}\right)$-cf. (29), using the complementary energy analogy the following replacements should be made in $(54)_{3}$,

$$
\frac{\left(\mathrm{k}_{\alpha}-\mathrm{k}_{\beta}\right)^{2} p^{2}}{\left(\mathrm{k}_{\alpha} \mathrm{k}_{\beta}\right)^{2}} \rightarrow \operatorname{tr}\left(\boldsymbol{\Gamma}_{\alpha}-\boldsymbol{\Gamma}_{\beta}\right), \quad \frac{\left(\mu_{\alpha}-\mu_{\beta}\right) \boldsymbol{\sigma}^{\prime} \cdot \boldsymbol{\sigma}^{\prime}}{\left(2 \mu_{\alpha} \mu_{\beta}\right)^{2}} \rightarrow \operatorname{dev}\left(\boldsymbol{\Gamma}_{\alpha}-\boldsymbol{\Gamma}_{\beta}\right) \cdot \operatorname{dev}\left(\boldsymbol{\Gamma}_{\alpha}-\boldsymbol{\Gamma}_{\beta}\right) .
$$

Then estimation of coherency energy can be expressed in the similar way as follows,

$$
\rho \phi^{\mathrm{coh}}=0.5 \sum_{\alpha, \beta=1, \beta \neq \alpha}^{n} z_{\alpha} z_{\beta} f_{\alpha \beta}\left(\boldsymbol{\Gamma}_{\alpha}, \boldsymbol{\Gamma}_{\beta}\right), \quad f_{\alpha \beta}\left(\boldsymbol{\Gamma}_{\alpha}, \boldsymbol{\Gamma}_{\beta}\right)=\frac{\mathrm{k}_{\alpha} \mathrm{k}_{\beta}\left(\gamma_{\alpha \beta}^{(v)}\right)^{2}}{2\left(\mathrm{k}^{V}+A^{(\mathrm{k})}\right)}+\frac{\mu_{\alpha} \mu_{\beta}\left(\gamma_{\alpha \beta}^{\prime}\right)^{2}}{\mu^{V}+A^{(\mu)}}
$$

where

$$
\gamma_{\alpha \beta}^{(v)} \equiv \operatorname{tr}\left(\boldsymbol{\Gamma}_{\alpha}-\boldsymbol{\Gamma}_{\beta}\right),\left(\gamma_{\alpha \beta}^{\prime}\right)^{2} \equiv \operatorname{dev}\left(\boldsymbol{\Gamma}_{\alpha}-\boldsymbol{\Gamma}_{\beta}\right) \cdot \operatorname{dev}\left(\boldsymbol{\Gamma}_{\alpha}-\boldsymbol{\Gamma}_{\beta}\right) .
$$

Furthermore, the estimates of phase averages of self-equilibrated internal stresses and macroscopic phase transition strains are found to be, cf. (23) and (26) 


$$
\begin{gathered}
\operatorname{dev} \boldsymbol{\sigma}_{\alpha}^{(\mathrm{in})}=\operatorname{dev} \Re_{\alpha}\left(\boldsymbol{\Gamma}_{\gamma}\right)=2 \mu_{\alpha} \sum_{\beta=1}^{n} \frac{z_{\beta} \mu_{\beta} \operatorname{dev}\left(\boldsymbol{\Gamma}_{\beta}-\boldsymbol{\Gamma}_{\alpha}\right)}{\mu^{V}+A^{(\mu)}} \\
\operatorname{tr} \boldsymbol{\sigma}_{\alpha}^{(\mathrm{in})}=\operatorname{tr} \mathfrak{\Re}_{\alpha}\left(\boldsymbol{\Gamma}_{\gamma}\right)=3 \mathrm{k}_{\alpha} \sum_{\beta=1}^{n} \frac{z_{\beta} \mathrm{k}_{\beta} \gamma_{\beta \alpha}^{(v)}}{\mathrm{k}^{V}+A^{(\mathrm{k})}} \\
\operatorname{dev} \boldsymbol{\varepsilon}^{\mathrm{pt}}=\sum_{\alpha=1}^{n} z_{\alpha} \operatorname{dev} \boldsymbol{\Gamma}_{\alpha}+\sum_{\alpha, \beta=1, \alpha \neq \beta}^{n} \frac{z_{\alpha} z_{\beta} \mu_{\beta} \operatorname{dev}\left(\boldsymbol{\Gamma}_{\beta}-\boldsymbol{\Gamma}_{\alpha}\right)}{\mu^{V}+A^{(\mu)}}, \quad \operatorname{dev} \boldsymbol{\varepsilon}^{\mathrm{pt}}=\mathbf{K} \boldsymbol{\varepsilon}^{\mathrm{pt}} \\
\operatorname{tr} \boldsymbol{\varepsilon}^{\mathrm{pt}}=\sum_{\alpha=1}^{n} z_{\alpha} \operatorname{tr}\left(\boldsymbol{\Gamma}_{\alpha}\right)+\sum_{\alpha, \beta=1, \alpha \neq \beta}^{n} \frac{z_{\alpha} z_{\beta} \mathrm{k}_{\beta} \gamma_{\beta \alpha}^{(v)}}{\mathrm{k}^{V}+A^{(\mathrm{k})}}, \quad \operatorname{tr} \boldsymbol{\varepsilon}^{\mathrm{pt}}=\mathbf{J} \boldsymbol{\varepsilon}^{\mathrm{pt}}
\end{gathered}
$$

The SEIM method, two parameter estimation of Gibbs free energy $g^{(V+A)}$ for isotropic RVE involving (52) becomes, cf. also (47),

$$
g^{(V+A)}=(\sum_{\alpha=1}^{n} z_{\alpha} \underbrace{g_{\alpha}^{0}}_{(47)_{2}})-\underbrace{g^{\mathrm{el}}}_{(54)_{1}}-\underbrace{\sigma \cdot \varepsilon^{\mathrm{pt}} / \rho}_{(59)}+\underbrace{\phi^{\mathrm{coh}}}_{(56)}
$$

Formula (60) is one of central results of the present work as it reveals explicit connections between microproperties, microstructure of SMA RVE and terms present in macroscopic Gibbs function for isotropic SMA macroelement composed of $n$ isotropic phases.

Now, let us solve (53) 2,3 with respect to $A^{(\mu)}$ and $A^{(\mathrm{k})}$

$$
\frac{1}{\mu^{V}+A^{(\mu)}}=\frac{\mu-\mu^{\mathrm{Re}}}{\mu\left[\mu^{V}-\mu^{\mathrm{Re}}\right]}, \quad \frac{1}{\mathrm{k}^{V}+A^{(\mathrm{k})}}=\frac{\mathrm{k}-\mathrm{k}^{\mathrm{Re}}}{\mathrm{k}\left[\mathrm{k}^{V}-\mathrm{k}^{\mathrm{Re}}\right]}
$$

By substituting relations (61) into (54), (56), (59) one can readily find the expressions for estimates of all principal properties of the isotropic RVE in terms of effective elastic moduli $\mu$ and k, i.e., $\boldsymbol{M}-\mathrm{cf}$. (53). When values of these two data are determined experimentally then all the other effective properties of isotropic, multiphase aggregate can be computed with the aid of derived above relations of two parameter, semiempirical variant of SEIM method. Relations derived in this section applied to isotropic SMA's with two-phase material microstructures $(n=2)$ lead to the same estimates as those obtained by Bernardini [2] with the following correspondence in notation $\left(c \leftrightarrow z, B \leftrightarrow A^{(\mathrm{k})}, A \leftrightarrow A^{(\mu)}\right)$. Thus, the present development can be regarded as generalization of two-phase proposal of Bernardini [2] to the case of microstructures with $n$ phase isotropic aggregates.

In Table 1 there are collected together values of $A^{(\mathrm{k})}, A^{(\mu)}$ parameters of SEIM method determined for some specific homogenization conjectures. The classical Voigt and Hashin-Shtrikman estimations are specified for two-phase aggregates.

It is interesting to point out here that conjectures made to obtain Hashin-Shtrikman estimates lead to mutual coupling of distortional and spherical deformation modes-judging on formulas in Table 1, a matter deserving separate investigation. This kind of coupling does not take place in the case of proposed here SEIM method of estimation.

\subsection{Two parameter estimation for isotropic aggregate of two isotropic phases $\left(\boldsymbol{A}_{\alpha \beta}=\boldsymbol{A}, n=2\right)$}

Let us track how coherence energy and macroscopic phase strain are parametrically influenced by the degree of inhomogeneity of elastic properties and magnitude of tensor $\boldsymbol{A}$. For that purpose let us accept the most common at present conjecture that SMA macroelement is in general a two-phase mixture of martensite and austenite, with microstructure and phase composition evolving in the course of isothermal processes of loading in temperature range of pseudoelastic behavior of the SMA material. The two-phase mixture is characterized by zero-phase eigenstrains in austenite $\left(\boldsymbol{\Gamma}_{1}=0\right)$, and deviatoric only phase eigenstrains in martensite $\left(\operatorname{dev} \boldsymbol{\Gamma}_{2}=\right.$ $\boldsymbol{\Gamma}, \operatorname{tr} \boldsymbol{\Gamma}_{2}=0$ ) - a typical modeling assumption for polycrystalline SMA materials. Thus, all volumetric effects are zero for investigated here case $\left(\gamma_{\alpha \beta}^{(v)}=0\right.$ - cf. $\left.(57)_{1}\right)$ and due to that only impact of $A^{(\mu)}$ needs to be 
Table 1 Summary of two parameter $\left(A^{(k)}, A^{(\mu)}\right)$ estimation of effective properties

\begin{tabular}{|c|c|c|c|c|}
\hline Estimation & $A^{(\mathrm{k})}$ & $A^{(\mu)}$ & $M$ cf.-(53) & $\rho \phi^{\mathrm{coh}}$ —cf. (56) \\
\hline Voigt & 0 & 0 & $\boldsymbol{M}^{V}$ & $\begin{array}{l}\sum_{\alpha, \beta=1, \beta \neq \alpha}^{n} \frac{1}{2} z_{\alpha} z_{\beta} \\
{\left[\frac{\mathrm{k}_{\alpha} \mathrm{k}_{\beta}\left(\gamma_{\alpha \beta}^{(v)}\right)^{2}}{2\left(\mathrm{k}^{V}+A^{(\mathrm{k})}\right)}\right.} \\
\left.+\frac{\mu_{\alpha} \mu_{\beta}\left(\gamma_{\alpha \beta}^{\prime}\right)^{2}}{\mu^{V}+A^{(\mu)}}\right]\end{array}$ \\
\hline $\begin{array}{l}\text { Hashin-Shtrikman lower } \\
\text { bound }(n=2)\end{array}$ & $\frac{3 \mathrm{k}_{1} \mathrm{k}_{2}}{4 \mu_{2}}$ & $\frac{6 \mu_{1}\left(\mathrm{k}_{2}+2 \mu_{2}\right)}{9 \mathrm{k}_{2}+8 \mu_{2}}$ & $\boldsymbol{M}=\frac{1}{3 \mathrm{k}} \mathbf{J}+\frac{1}{2 \mu} \mathbf{K}$ & \\
\hline $\begin{array}{l}\text { Semiexperimental } \\
\text { SEIM method }\end{array}$ & $A_{\exp }^{(\mathrm{k})}$ & $\boldsymbol{A}_{\mathrm{exp}}^{(\mu)}$ & $\mathrm{k}=\mathrm{k}^{\operatorname{Re}} \frac{\mathrm{k}^{V}+A^{(\mathrm{k})}}{\mathrm{k}^{\operatorname{Re}}+A^{(\mathrm{k})}}$ & \\
\hline $\begin{array}{l}\text { Hashin-Shtrikman upper } \\
\text { bound }(n=2)\end{array}$ & $\frac{3 k_{1} k_{2}}{4 \mu_{1}}$ & $\frac{6 \mu_{2}\left(\mathrm{k}_{1}+2 \mu_{1}\right)}{9 \mathrm{k}_{1}+8 \mu_{1}}$ & $\mu=\mu^{\operatorname{Re}} \frac{\mu^{V}+A^{(\mu)}}{\mu^{\operatorname{Re}}+A^{(\mu)}}$ & \\
\hline Reuss & $\infty$ & $\infty$ & $\boldsymbol{M}^{\mathrm{Re}}$ & 0 \\
\hline
\end{tabular}

studied. The estimations of coherence energy and macroscopic phase strain can be straightforwardly obtained upon use of (56) and (59) for $n=2$. However, for the present purposes it is convenient to use normalized estimations defined as follows,

$$
y \equiv \frac{\rho \phi^{\mathrm{coh}}}{\left(\Gamma_{\mathrm{eq}} \cdot \mu^{G} \Gamma_{\mathrm{eq}}\right)}=z_{1} z_{2} \frac{(3 / 2) \mu^{G}}{\left[z_{1} \mu_{1}+z_{2} \mu_{2}+A^{(\mu)}\right]}, \quad \frac{\varepsilon_{\mathrm{eq}}^{\mathrm{pt}}}{\Gamma_{\mathrm{eq}}}=\frac{z_{2}\left(\mu_{2}+A^{(\mu)}\right)}{\left(1-z_{2}\right) \mu_{1}+z_{2} \mu_{2}+A^{(\mu)}} .
$$

The symbol $\mu^{G}$ denotes the geometric mean of shear moduli, $\varepsilon_{\mathrm{eq}}^{\mathrm{pt}}$ is the equivalent macroscopic phase strain, and $\Gamma_{e f}$ is the equivalent microeigenstrain of martensite,

$$
\mu^{G} \equiv \sqrt{\mu_{1} \mu_{2}}, \quad \mu_{12} \equiv \mu_{1} / \mu_{2}, \quad \boldsymbol{\varepsilon}_{\mathrm{eq}}^{\mathrm{pt}}=\sqrt{\frac{2}{3} \boldsymbol{\varepsilon}^{\prime \mathrm{pt}} \cdot \boldsymbol{\varepsilon}^{\prime \mathrm{pt}}}, \quad \boldsymbol{\Gamma}_{\mathrm{eq}} \equiv \sqrt{\frac{2}{3} \operatorname{dev} \boldsymbol{\Gamma} \cdot \operatorname{dev} \boldsymbol{\Gamma}} .
$$

The dependence of $y$ on shear moduli and $z_{2}$ is invariant under simultaneous replacement of $\mu_{1} \leftrightarrow \mu_{2}$ and $\left(z_{2}-0.5\right) \leftrightarrow\left(0.5-z_{2}\right)$. Figure 1 shows variation of the normalized (dimensionless) coherency energy $y$ with changing volume fraction of martensitic phase $z_{2}$ for four values of the elastic shear moduli ratio $\mu_{12} \equiv \mu_{1} / \mu_{2},(0.5,1,2,4)$ and for two values of parameter $A^{(\mu)},(0,10)$. The Voigt-type, an upper bound, estimates are drawn with dashed lines $\left(A^{(\mu)}=0\right)$. The solid lines show the dependence of the coherency energy on $z_{2}$ for $A^{(\mu)} / \mu^{G}=10$. The solid curves obtained for different values of the ratio $\mu_{12}$ are practically indistinguishable in the scale adopted in Fig. 1. The maximum values of normalized coherency energy and coordinates of their location are

$$
\begin{gathered}
\left.y_{\max } \equiv \frac{\rho \phi^{\mathrm{coh}}}{\mu^{G}\left(\boldsymbol{\Gamma}_{\mathrm{eq}}\right)^{2}}\right|_{z_{2}=z_{2} \max }=\frac{3}{2} \mu^{G}\left[\frac{\sqrt{\mu_{1}+A^{(\mu)}}-\sqrt{\mu_{2}+A^{(\mu)}}}{\mu_{1}-\mu_{2}}\right]^{2}, \\
z_{2 \max }-0.5=\frac{\sqrt{\mu_{1}+A^{(\mu)}}-\sqrt{\mu_{2}+A^{(\mu)}}}{2\left[\sqrt{\mu_{1}+A^{(\mu)}}+\sqrt{\mu_{2}+A^{(\mu)}}\right]},\left.\quad y_{1 \max } \equiv y_{\max }\right|_{\mu_{1}=\mu_{2}=\mu^{G}}=\frac{3}{8\left(1+A^{(\mu)} / \mu^{G}\right)} .
\end{gathered}
$$

The highest peak among the Voigt-type estimates maxima of the coherency energy is denoted $y_{1 \text { max }}\left(y_{1 \max } \geq\right.$ $\left.\left.y_{\max }\right|_{\mu_{1} \neq \mu_{2}}\right)$. It arises when there is no microscopic heterogeneity of elastic properties $\left(\mu_{1}=\mu_{2}\right)$.

Let us define a dimensionless parameter $\Delta y \equiv\left(y_{1 \max } / y_{\max }\right)-1$. It makes convenient measure of the sensitivity of the coherency energy to the microscopic heterogeneity of elastic properties. The parameter $\Delta y$ is also invariant under the transformation $\mu_{1} \leftrightarrow \mu_{2}$. For $A^{(\mu)}=0$ it is equal to $0.25\left(\sqrt{\mu_{12}}-1\right)^{2} / \sqrt{\mu_{12}}$. Plots of $\Delta y$ versus $A^{(\mu)} / \mu^{G}$ for two values of $\mu_{12} \equiv \mu_{1} / \mu_{2},(2,4)$ are shown in Fig. 2a. A similar to $\Delta y$ dimensionless parameter of elastic heterogeneity sensitivity can also be defined for macroscopic phase strain. Using (62) one finds the following connection $\varepsilon_{\mathrm{eq}}^{\mathrm{pt}}-z_{2} \Gamma_{\mathrm{eq}}=2 \Gamma_{\mathrm{eq}}\left(\mu_{2}-\mu_{1}\right) y / 3 \mu^{G}$. Hence,

$$
\Delta y \equiv\left(y_{1 \max } / y_{\max }\right)-1, \quad \Delta e \equiv \frac{2}{3} y_{\max }\left|\mu_{2}-\mu_{1}\right| / \mu^{G}, \quad\left|\varepsilon_{\mathrm{eq}}^{\mathrm{pt}}-z_{2} \Gamma_{\mathrm{eq}}\right| \leq \Gamma_{\mathrm{eq}} \Delta e
$$




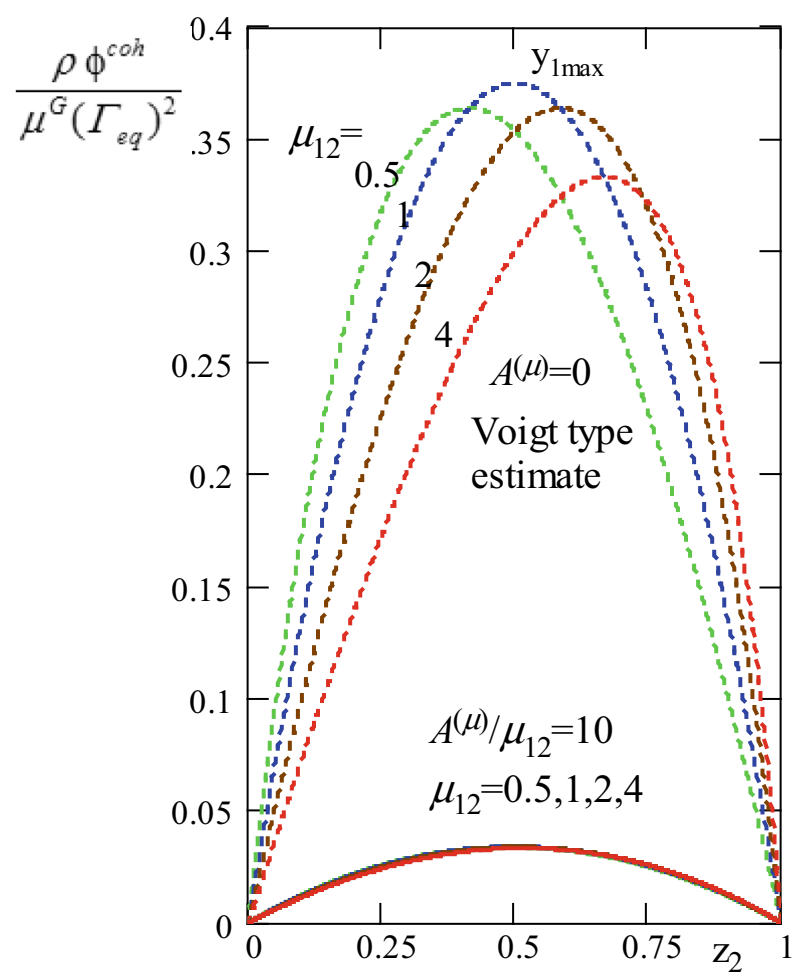

Fig. 1 The dependence of normalized (dimensionless) coherency energy $y$ on the martensite volume fraction $z_{2}$ for several values of parameters $\mu_{12}(0.5,1,2,4)$ and $A^{(\mu)} / \mu^{G}(0,10)$

(a)

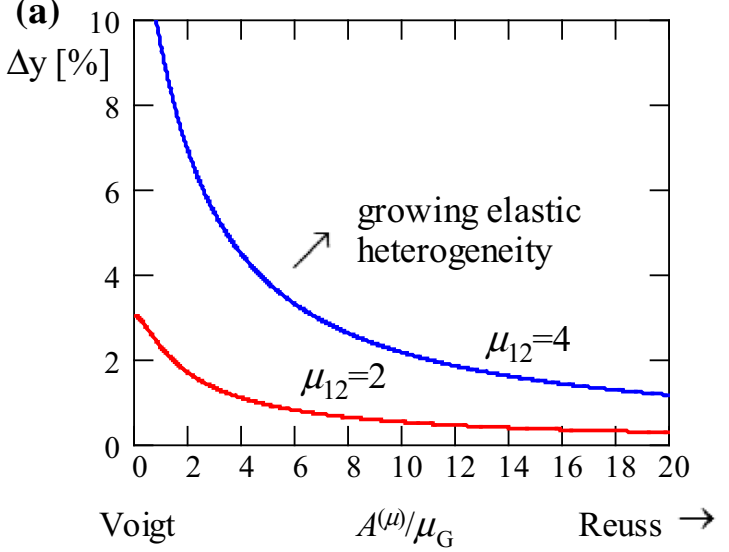

(b)

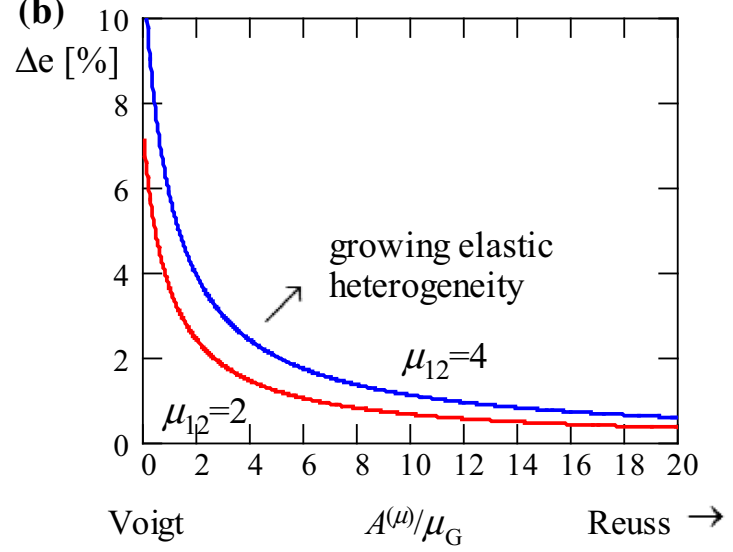

Fig. 2 a) Variation of the relative error $\Delta y[\%]$-in estimation of the coherency energy, with the value of ratio $A^{(\mu)} / \mu^{G}$, b) Variation of the relative error $\Delta e[\%]$-in estimation of the equivalent macroscopic phase strain $\varepsilon_{\mathrm{eq}}^{\mathrm{pt}}$, with the value of ratio $A^{(\mu)} / \mu^{G}$

The parameter $\Delta e$ is invariant under mutual change of the shear moduli $\mu_{1} \leftrightarrow \mu_{2}$. The variations of $\Delta e$ with $A^{(\mu)} / \mu^{G}$ for $\mu_{12}=2$ and $\mu_{12}=4$ are shown in Fig. $2 \mathrm{~b}$.

Naturally both measures $\Delta y$ and $\Delta e$ of elastic heterogeneity sensitivities vanish when the shear moduli of the phases are the same.

To find the order of magnitude of realistic values of the modulus $A^{(\mu)}$ a key presumption underlying the family of two-phase SMA material models-recalled in Sect. 2, is used stating that phase transitions are initiated at unstable thermodynamically phase equilibria states. This simultaneously means that phase transition criteria as well as the landscapes of phase equilibria in diverse diagrams are derivable directly from the adopted form of thermodynamic potential. The forward and reverse martensitic phase transitions are initiated when 
thermodynamic driving force is equal to zero. Let us specify the criteria for start of martensitic transformations from pure austenite and/or pure martensite in the conditions of isothermal, uniaxial tension loading. In such a case thermodynamic driving force of phase transition (4) takes the form,

$$
\left.\begin{array}{l}
\pi^{1-2}\left(\sigma_{\text {ten }}^{\mathrm{AM}}, T, z=0\right)=\left(\pi_{0}^{f}-\phi_{2}^{\mathrm{st}}\right)-\phi_{i t}+\gamma_{\text {ten }} \cdot \sigma_{\text {ten }}^{\mathrm{AM}} / \rho=0 \\
\pi^{1-2}\left(\sigma_{\text {ten }}^{\mathrm{MA}}, T, z=1\right)=\left(\pi_{0}^{f}-\phi_{2}^{\mathrm{st}}\right)+\phi_{i t}+\gamma_{\text {ten }} \cdot \sigma_{\text {ten }}^{\mathrm{MA}} / \rho=0
\end{array}\right\} \Rightarrow 2 \rho \phi_{i t}=\left(\sigma_{\text {ten }}^{\mathrm{AM}}-\sigma_{\text {ten }}^{\mathrm{MA}}\right) \gamma_{\text {ten }}
$$

The symbol $\sigma_{\text {ten }}^{\mathrm{AM}}$ denotes the critical uniaxial stress at which the pseudoelastic flow starts for pure austenite, whereas $\sigma_{\text {ten }}^{\mathrm{MA}}$ denotes the critical uniaxial stress at which the decomposition of pure martensite begins in an isothermal, simple tension test at some temperature $T$ above $A_{f}^{0}$ temperature. Symbol $\gamma_{\text {ten }}$ denotes pseudoelastic flow amplitude of uniaxial strain connected with full forward $(z=0 \rightarrow 1)$ and/or full reverse $(z=1 \rightarrow 0)$ phase transition in simple tension. Upon elimination of the term $\left(\pi_{0}^{f}-\phi_{2}^{\text {st }}\right)$ from relations $(66)_{1}$, relation $(66)_{2}$ is obtained. It is easy to notice that the following equivalence in denotations is valid here $\phi_{i t}=\phi_{12}^{(0) \text { coh }}$. Please note that whenever ideal pseudoelastic flow takes place for specific SMA material then the difference $\left(\sigma_{\text {ten }}^{\mathrm{AM}}-\sigma_{\mathrm{ten}}^{\mathrm{MA}}\right)$ determines the width of outermost pseudoelastic hysteresis loop. Within the same assumption $2 \rho \phi_{12}^{(0) c o h}$ determines area of hysteresis loop in stress-strain coordinates, upon relation $(66)_{2}$.

The data for polycrystalline $\mathrm{Ni}_{51 \mathrm{at} \%}-\mathrm{Ti}$ alloy obtained in isothermal experiments performed in the temperature range of pseudoelasticity suggest that $\mu_{1}=\mu_{2}=\mu^{G} \approx 20 \times 10^{3}[\mathrm{MPa}$ ] (homogeneous elastic properties $), \gamma_{\text {ten }} \approx 0.06$ and $\left(\sigma_{\text {ten }}^{\mathrm{AM}}-\sigma_{\text {ten }}^{\mathrm{MA}}\right) \approx 200$ [MPa] cf. [24]. Substitution of this values to $(66)_{2}$ gives the approximate value of $\rho \phi_{12}^{(0) \text { coh }}$ to be about $6[\mathrm{MPa}$. When elastic properties of SMA macroelement can be accepted as homogeneous, then the following formula is valid $\rho \phi_{12}^{(0) \text { coh }}=\mu^{G} \frac{3}{2}\left(\gamma_{\text {ten }}\right)^{2} /\left(1+A^{(\mu)} / \mu^{G}\right)$ upon $(67)_{2}$ with $\left(\gamma_{\alpha \beta}^{(v)}=0, \gamma_{\alpha \beta}^{\prime}=\gamma_{\text {ten }}\right)$. This for $\rho \phi_{12}^{(0) \text { coh }} \cong 6[\mathrm{MPa}]$ implies $A^{(\mu)} / \mu^{G} \cong 17$, viz. the order of magnitude of $A^{(\mu)}$ is $10 \mu^{G}$. Within the assumptions of the present section, i.e., with stored coherency energy equal to zero $\left(\phi_{2}^{\text {st }}=0\right)$, the maximum value of coherency energy $\rho \phi_{\max }^{\text {coh }}=\rho \phi^{\text {coh }}\left(z_{1,2}=\frac{1}{2} ; \mu_{1}=\mu_{2}\right)$ reaches the value $\phi_{\max }^{\text {coh }}=\frac{1}{4} \phi_{12}^{(0) \text { coh }} \cong 1.5[\mathrm{MPa}]$, in accordance with $(67)_{1}\left(\rho \phi^{\text {coh }}=z_{1} z_{2} \rho \phi_{12}^{(0) \text { coh }}\right)$.

From this, it is clear that the Voigt estimates considerably exceed the actual coherency energy. The second information is that the high value of the actual estimation of the modulus $A^{(\mu)}$ for NiTi alloy $\left(A^{(\mu)} / \mu^{G} \cong 17\right)$, of the order of magnitude higher than the value of estimation of shear modulus, proves that the heterogeneity of microscopic elastic properties of the two-phase isotropic mixture has no essential influence on the value of coherency energy for this alloy. Both these properties are well illustrated in Fig. 1.

Returning to sensitivity charts shown in Fig. 2. It is seen that even for the relatively high value $\mu_{12}=4$ the assumption $\mu_{1}=\mu_{2}=\mu^{G}$ leads to the error lesser than $2.5 \%(\Delta y)$ in evaluation of the coherency energy, provided that $A^{(\mu)} / \mu^{G} \geq 10$. Similarly for $A^{(\mu)} / \mu^{G} \geq 10$ the relative error $(\Delta e)$ in estimation of the equivalent macroscopic phase strain following from disregarding the microscopic heterogeneity is less than $2 \%$. Thus, it is seen that even when elastic properties of specific SMA material are relatively inhomogeneous, neglecting this fact in evaluation of the coherency energy or macroscopic phase strain does not lead to considerable errors in their values estimations.

Bernardini [2] used quite different rather unusual values of the elastic shear moduli for the two-phase NiTi alloy (e.g., $\mu_{1}=11278[\mathrm{MPa}], \mu_{12} \simeq 2.307$ ). Among the other things, he calculated the Hashin-Shtrikman (HS) upper and lower bounds and showed that the micromechanics-based theory presented in [27] largely overestimates the coherency energy. When the data presented in [2] are used it is found that the ratio $A^{(\mu)} / \mu^{G}$ is approximately 1.33 and 0.58 for the lower and upper HS bounds, respectively. Even for so low values of the ratio $A^{(\mu)} / \mu^{G}$, the relative error in evaluation of the coherency energy is less than $4 \%$. They overestimate the maximum coherency energy and the value of $\rho \phi_{12}^{(0) \text { coh }}$ found here. The application of those values would entail the prediction of too large thickness of the pseudoelastic hysteresis loop, not observed experimentally.

5.3 Estimation for isotropic aggregate of $n$ isotropic phases with no elastic heterogeneity $\left(\boldsymbol{M}_{\alpha}=\boldsymbol{M}\right)$

When all phases have the same elastic constants $\left(\mu^{V}=\mu^{\mathrm{Re}}=\mu_{\alpha}=\mu, \mathrm{k}^{V}=\mathrm{k}^{\mathrm{Re}}=\mathrm{k}_{\alpha}=\mathrm{k}, g_{\alpha \beta}^{\mathrm{el}}=0\right)$ as for elastically homogeneous SMA material $\mathfrak{\Im}_{\alpha \beta}=\boldsymbol{L}-\mathrm{cf}$. (13) $)_{1}$. Then, expressions for the coherency energy coefficients, the phase averages of self-equilibrated stresses and macroscopic phase strains reduce to 


$$
\begin{gathered}
\rho \phi^{\mathrm{coh}}=\frac{1}{2} \sum_{\alpha, \beta=1, \beta \neq \alpha}^{n} z_{\alpha} z_{\beta} \rho \phi_{\alpha \beta}^{(0) \mathrm{coh}}, \quad \rho \phi_{\alpha \beta}^{(0) \operatorname{coh}}=\frac{k\left(\gamma_{\alpha \beta}^{(v)}\right)^{2}}{2\left[1+A^{(\mathrm{k})} / \mathrm{k}\right]}+\frac{\mu\left(\gamma_{\alpha \beta}^{\prime}\right)^{2}}{\left[1+A^{(\mu)} / \mu\right]}, \\
\boldsymbol{\varepsilon}^{\mathrm{pt}}=\langle\boldsymbol{\Gamma}\rangle_{V} \equiv \sum_{\alpha=1}^{n} z_{\alpha} \boldsymbol{\Gamma}_{\alpha}, \operatorname{dev} \boldsymbol{\sigma}_{\alpha}^{(\mathrm{in})}=\frac{2 \mu\left[\langle\operatorname{dev} \boldsymbol{\Gamma}\rangle_{V}-\operatorname{dev} \boldsymbol{\Gamma}_{\alpha}\right]}{1+A^{(\mu)} / \mu}, \operatorname{tr} \boldsymbol{\sigma}_{\alpha}^{(\mathrm{in})}=\frac{3 \mathrm{k}\left[\langle\operatorname{tr} \boldsymbol{\Gamma}\rangle_{V}-\operatorname{tr} \boldsymbol{\Gamma}_{\alpha}\right]}{1+A^{(\mathrm{k})} / \mathrm{k}} .
\end{gathered}
$$

In this situation the coherency energy coefficients may depend on $z_{\alpha}$ only through possible dependence of $A^{(\mu)}$ and $A^{(k)}$ on phase composition. The $\gamma_{\alpha \beta}^{\prime}$ is defined in (57).

\section{Stored coherency energy and fluctuating part of microeigenstrain field}

In evaluating the significance of fluctuating parts of eigenstrains fields $\breve{\boldsymbol{\Gamma}}_{\alpha}^{f}(\boldsymbol{x})$ on free energy terms let us return to general case of $n$ phase RVE discussed in Sect. 4. The nonzero fluctuating part of microeigenstrain field $\breve{\boldsymbol{\Gamma}}_{\alpha}^{f}(\boldsymbol{x}) \neq 0$-cf. (10), has no influence on the distribution of external fields in multiphase macroelement. Since the distribution of elastic compliances was presumed to be piecewise constant all general expressions derived in Sects. 4, 5 and concerning averaged quantities of external fields and macroscopic elastic complementary energy $g^{\text {el }}$-cf. (27), remain valid.

The expressions concerning internal fields including the macroscopic phase strain must be modified. The approach discussed in Sects. 4.1 and 4.2 can still be used but in (12) 4 to the phase averages $\boldsymbol{\Gamma}_{\alpha}$ there must be added nonzero at present phase fluctuating parts $\breve{\boldsymbol{\Gamma}}_{\alpha}^{f}(\boldsymbol{x}) \neq 0$ of $\breve{\boldsymbol{\Gamma}}_{\alpha}(\boldsymbol{x}) \quad\left(\boldsymbol{x} \in V_{\alpha}, \alpha=1, \ldots, n\right)$. Next internal fields problem is additively divided into the fields generated by phase averages and phase fluctuations of meso-eigenstrains. The former will be denoted by superscript ${ }^{(\mathrm{a}-\mathrm{in})}$ and the latter by superscript ${ }^{(\mathrm{f}-\mathrm{in})}$

$$
\breve{\boldsymbol{\varepsilon}}_{\alpha}^{(\mathrm{in})}(\boldsymbol{x})=\breve{\boldsymbol{\varepsilon}}_{\alpha}^{(\mathrm{a}-\mathrm{in})}(\boldsymbol{x})+\breve{\boldsymbol{\varepsilon}}_{\alpha}^{(\mathrm{f}-\mathrm{in})}(\boldsymbol{x}), \quad \breve{\boldsymbol{\sigma}}_{\alpha}^{(\mathrm{in})}(\boldsymbol{x})=\breve{\boldsymbol{\sigma}}_{\alpha}^{(\mathrm{a}-\mathrm{in})}(\boldsymbol{x})+\breve{\boldsymbol{\sigma}}_{\alpha}^{(\mathrm{f}-\mathrm{in})}(\boldsymbol{x}), \quad \boldsymbol{x} \in V_{\alpha}
$$

These fields are supposed to fulfill, in each local volume $x \in V_{\alpha}$, the constitutive relations

$$
\breve{\boldsymbol{\varepsilon}}_{\alpha}^{(\mathrm{a}-\mathrm{in})}(\boldsymbol{x})=\boldsymbol{M}_{\alpha} \breve{\boldsymbol{\sigma}}_{\alpha}^{(\mathrm{a}-\mathrm{in})}(\boldsymbol{x})+\boldsymbol{\Gamma}_{\alpha}, \quad \breve{\boldsymbol{\varepsilon}}_{\alpha}^{(\mathrm{f} \text {-in })}(\boldsymbol{x})=\boldsymbol{M}_{\alpha} \breve{\boldsymbol{\sigma}}_{\alpha}^{(\mathrm{f}-\mathrm{in})}(\boldsymbol{x})+\breve{\boldsymbol{\Gamma}}_{\alpha}^{f}(\boldsymbol{x}), \quad(! \alpha)
$$

Both fields $\breve{\boldsymbol{\sigma}}_{\alpha}^{(\mathrm{a}-\mathrm{in})}$ and $\breve{\boldsymbol{\sigma}}_{\alpha}^{(\mathrm{f} \text {-in) }}(\boldsymbol{x})$ are self-equilibrated stress fields, and phase averages of $\breve{\boldsymbol{\Gamma}}_{\alpha}^{f}(\boldsymbol{x})$ vanish $\left\langle\breve{\boldsymbol{\Gamma}}_{\alpha}^{f}(\boldsymbol{x})\right\rangle_{V_{\alpha}}=0,(\alpha=1, \ldots, n)$-cf. (10). The constitutive relations between phase averages of the local fields $\boldsymbol{\varepsilon}_{\alpha}^{(\mathrm{a}-\mathrm{in})} \equiv\left\langle\overline{\boldsymbol{\varepsilon}}^{(\mathrm{a}-\mathrm{in})}(\boldsymbol{x})\right\rangle_{V_{\alpha}}, \boldsymbol{\varepsilon}_{\alpha}^{(\mathrm{f}-\mathrm{in})}=\left\langle\widetilde{\boldsymbol{\varepsilon}}^{(\mathrm{a}-\mathrm{in})}(\boldsymbol{x})\right\rangle_{V_{\alpha}}, \boldsymbol{\sigma}_{\alpha}^{(\mathrm{a}-\mathrm{in})}=\left\langle\widehat{\boldsymbol{\sigma}}^{(\mathrm{a}-\mathrm{i})}(\boldsymbol{x})\right\rangle_{V_{\alpha}}, \boldsymbol{\sigma}_{\alpha}^{(\mathrm{f}-\mathrm{in})}=\left\langle\widehat{\boldsymbol{\sigma}}^{(\mathrm{f}-\mathrm{in})}(\boldsymbol{x})\right\rangle_{V_{\alpha}}$ in each volume $V_{\alpha}$ are

$$
\boldsymbol{\varepsilon}_{\alpha}^{(\mathrm{a}-\mathrm{in})}=\boldsymbol{M}_{\alpha} \boldsymbol{\sigma}_{\alpha}^{(\mathrm{a}-\mathrm{in})}+\boldsymbol{\Gamma}_{\alpha}, \boldsymbol{\varepsilon}_{\alpha}^{(\mathrm{f}-\mathrm{in})}=\boldsymbol{M}_{\alpha} \boldsymbol{\sigma}_{\alpha}^{(\mathrm{f}-\mathrm{in})}, \sum_{\alpha=1}^{n} z_{\alpha} \boldsymbol{\sigma}_{\alpha}^{(\mathrm{a}-\mathrm{in})}=0, \sum_{\alpha=1}^{n} z_{\alpha} \boldsymbol{\sigma}_{\alpha}^{(\mathrm{f}-\mathrm{in})}=0
$$

The formal connections derived in Sect. 4 linking $\boldsymbol{\sigma}_{\alpha}^{(\mathrm{in})}$ and $\boldsymbol{\varepsilon}_{\alpha}^{(\mathrm{in})}$ with $z_{\alpha}, \boldsymbol{\Gamma}_{\alpha}$ and ingredients of phase concentration moduli such as (23) $)_{1}$ and (26) 2 are now valid, only with reference to $\boldsymbol{\sigma}_{\alpha}^{(\mathrm{a}-\mathrm{in})}$ and $\boldsymbol{\varepsilon}_{\alpha}^{(\mathrm{a}-\mathrm{in})}$. It is sufficient to substitute $\boldsymbol{\sigma}_{\alpha}^{(\mathrm{a}-\mathrm{in})}$ and $\boldsymbol{\varepsilon}_{\alpha}^{(\mathrm{a}-\mathrm{in})}$ in place of $\boldsymbol{\sigma}_{\alpha}^{(\mathrm{in})}$ and $\boldsymbol{\varepsilon}_{\alpha}^{(\mathrm{in})}$ in corresponding formulas presented in Sect. 4.

The macroscopic phase strain is influenced by $\boldsymbol{\varepsilon}_{\alpha}^{(\mathrm{f}-\mathrm{in})}$ when some phases have different elastic compliances, otherwise, $\boldsymbol{\varepsilon}^{\mathrm{pt}}=\sum_{\alpha=1}^{n} z_{\alpha} \boldsymbol{\Gamma}_{\alpha}$.

$$
\begin{aligned}
\boldsymbol{\varepsilon}^{\mathrm{pt}} & =\sum_{\alpha=1}^{n} z_{\alpha} \boldsymbol{\varepsilon}_{\alpha}^{(\mathrm{in})}, \quad \boldsymbol{\varepsilon}_{\alpha}^{(\mathrm{in})}=\boldsymbol{M}_{\alpha}\left(\boldsymbol{\sigma}_{\alpha}^{(\mathrm{a}-\mathrm{in})}+\boldsymbol{\sigma}_{\alpha}^{(\mathrm{f}-\mathrm{in})}\right)+\boldsymbol{\Gamma}_{\alpha}, \\
\boldsymbol{\varepsilon}_{\alpha}^{(\mathrm{in})} & =\boldsymbol{\Gamma}_{\alpha}+\sum_{\beta=1, \beta \neq \alpha}^{n} z_{\beta} \boldsymbol{M}_{\alpha}\left[\Im_{\alpha \beta}^{(S)}\left(\boldsymbol{\Gamma}_{\beta}-\boldsymbol{\Gamma}_{\alpha}\right)+\Im_{\alpha \beta}^{(A)} \boldsymbol{\Gamma}_{\beta}\right]+\boldsymbol{\varepsilon}_{\alpha}^{(\mathrm{f}-\mathrm{in})}
\end{aligned}
$$


The alternative decomposition of $\boldsymbol{\varepsilon}^{\mathrm{pt}}$ corresponding to (26) 2 now takes the form

$$
\boldsymbol{\varepsilon}^{\mathrm{pt}}=z_{1} \boldsymbol{\Gamma}_{1}+\sum_{\alpha=2}^{n} z_{\alpha} \boldsymbol{\kappa}_{\alpha}, \quad \boldsymbol{\kappa}_{\alpha} \equiv \boldsymbol{\Gamma}_{\alpha}+\left(\boldsymbol{M}_{\alpha}-\boldsymbol{M}_{1}\right) \boldsymbol{L}_{\alpha}\left(\boldsymbol{\varepsilon}_{\alpha}^{(\mathrm{in})}-\boldsymbol{\Gamma}_{\alpha}\right), \quad(! \alpha)
$$

To derive modified formal expression for coherency energy we shall utilize the usual elastic reciprocal theorem which in the present context reads

$$
\sum_{\alpha=1}^{n} z_{\alpha} \boldsymbol{\Gamma}_{\alpha} \cdot \boldsymbol{\sigma}_{\alpha}^{(\mathrm{f}-\mathrm{in})}=\sum_{\alpha=1}^{n} z_{\alpha}\left\langle\breve{\boldsymbol{\Gamma}}^{f}(\boldsymbol{x}) \breve{\boldsymbol{\sigma}}^{(\mathrm{a}-\mathrm{in})}(\boldsymbol{x})\right\rangle_{V_{\alpha}}
$$

By combining (9) 2 with (10) 1 and (69) 2 , and next using (74), after some algebraic operations, one finds the following general form for the coherency energy

$$
\begin{aligned}
\phi^{\mathrm{coh}} & =\phi^{(a) \mathrm{coh}}+\phi^{(\mathrm{a}-\mathrm{f}) \mathrm{coh}}+\phi^{(f) \mathrm{coh}} \geq 0, \\
\rho \phi^{(a) \mathrm{coh}} & =0.5 \sum_{\alpha=1}^{n} z_{\alpha} \boldsymbol{\Gamma}_{\alpha} \cdot\left(-\boldsymbol{\sigma}^{(\mathrm{a}-\mathrm{in})}\right), \quad \phi^{(a) \mathrm{coh}} \equiv 0.5 \sum_{\alpha, \beta=1, \alpha \neq \beta}^{n} z_{\alpha} z_{\beta} \phi_{\alpha \beta}^{(0) \mathrm{coh}}, \\
\rho \phi^{(a-f) \mathrm{coh}} & =-0.5 \sum_{\alpha, \beta=1, \alpha \neq \beta}^{n} z_{\alpha} z_{\beta}\left[\left(\boldsymbol{\Gamma}_{\alpha}-\boldsymbol{\Gamma}_{\beta}\right) \cdot \boldsymbol{\sigma}_{\alpha}^{(\mathrm{f}-\mathrm{in})}+\left(\boldsymbol{\Gamma}_{\beta}-\boldsymbol{\Gamma}_{\alpha}\right) \cdot \boldsymbol{\sigma}_{\beta}^{(\mathrm{f}-\mathrm{in})}\right], \\
\rho \phi^{(f) \mathrm{coh}} & =\sum_{\alpha=1}^{n} z_{\alpha} \rho \phi_{\alpha}^{\mathrm{coh}}, \quad \rho \phi_{\alpha}^{\mathrm{coh}}=0.5\left\langle\breve{\boldsymbol{\Gamma}}^{f}(\boldsymbol{x}) \cdot\left(-\breve{\boldsymbol{\sigma}}^{(\mathrm{f}-\mathrm{in})}(\boldsymbol{x})\right)\right\rangle_{V_{\alpha}} \geq 0 .
\end{aligned}
$$

Please note that the actual proposal for coherence energy in the form $\phi^{\mathrm{coh}}=\phi_{2}^{\mathrm{st}} z_{2}+\phi_{i t} z_{1} z_{2}-\mathrm{cf}$. (1), is consistent with the above formula. The $\phi^{(a) c o h}$ and $\phi^{(f) c o h}$ are coherency energies generated by separate action of average $\boldsymbol{\Gamma}_{\alpha}$ and fluctuation $\breve{\boldsymbol{\Gamma}}_{\alpha}^{f}(\boldsymbol{x})$ eigenstrains, respectively. The general expression of the earlier energy in terms of ingredients of stress concentration moduli is given in Sect. 4.2 by (29)—cf. also (21). The term $\phi^{(a-f) c o h}$, linear in $\boldsymbol{\Gamma}_{\alpha}$ and semiquadratic in phase composition, reflects the influence of coupling between the fields $\breve{\boldsymbol{\sigma}}^{(\mathrm{a} \text {-in) }}(\boldsymbol{x})$ and $\breve{\boldsymbol{\sigma}}^{\text {(f-in) }}(\boldsymbol{x})$ on the coherency energy. The $\phi^{(a-f) \operatorname{coh}}$ vanishes, similarly to $\phi^{(a) \text { coh }}$, when the austenite is totally decomposed into a single martensitic compound, viz., $\left.\phi^{(a-f) \operatorname{coh}}\right|_{z_{\alpha}=1}=0$. This property is not shared by $\phi^{(f)}$ coh term. After completion of decomposition of austenite into the single martensitic compound the term $\phi^{(f) \text { coh }}$ represents the energy stored in RVE due to the presence of residual self-equilibrated

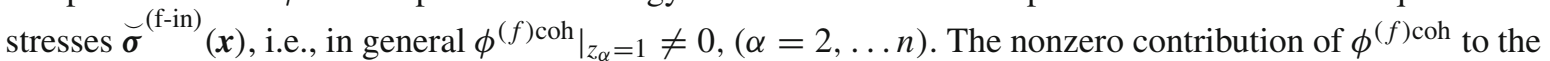
coherency energy is essential in the explanation of the macroscopic behavior effect observed experimentally for NiTi alloys. Namely, the characteristic start temperature of reverse austenitic phase transition $A_{s}^{0}$ at zero assisting macroscopic stress of NiTi alloy not submitted to mechanical loading-unloading cycle in martensitic state, at low temperature, is considerably different from start temperature of reverse transition, let us call it $A_{s}^{2-1}\left(A_{s}^{2-1}>A_{s}^{0}\right)$ of the same NiTi material submitted to high mechanical loading cycle in martensitic state at low temperatures, cf. also Popov and Lagoudas [19] and chapter 3 in Ziolkowski [32]. The appearance of this effect itself delivers experimental premise for presence of nonzero, incompatible, phase fluctuation fields $\left(\breve{\boldsymbol{\Gamma}}^{f}(\boldsymbol{x}) \neq 0\right)$ —cf. $(75)_{2}$ in NiTi alloys, induced by phase transitions resulting from thermomechanical loadings.

\section{Concluding remarks}

Using selected ideas of the homogenization theory of thermoelastic materials a new method for consistent estimation of the complementary elastic energy, coherency energy and phase transition strains of n-phase mixture RVE of SMA material has been proposed. Restricting considerations to uniform traction boundary conditions-not a critical limiting assumption, the coefficients appearing in relevant expressions for the mentioned objects have been expressed in terms of symmetric and skew-symmetric ingredients of self-equilibrated eigenstrains influence moduli (SEIM) by (27), (29) (26) 1,2 . The derived final expressions, in the form called 
here semilinear and semiquadratic functions of phase compositions, are most adequate in situations when large fractions of parent phase decompose into other phases. The advantages following from application of such a form in the range of pseudoelastic behavior of SMA materials may be found in several papers. However, it seems that the interpretation of involved coefficients in terms of moduli of stress concentration constitutes a novelty in the technical literature devoted to SMA. It has been shown that the same array of scalar functions defines the coefficients $g_{\alpha \beta}^{\mathrm{el}}$ of macroscopic elastic complementary energy and the coefficients of coherency energy $\phi_{\alpha \beta}^{(0) c o h}$, provided that a mixture of $n$-solid phases at microscale is characterized by piecewise uniform distributions of elastic compliances and phase eigenstrains. Thus, clear interpretation of modeling terms present in SMA material Gibbs free energy function in terms of actual physical objects has been reached. Using experimental data it has been shown that the coherency energy $\phi^{\text {coh }}$ of NiTi isotropic alloys treated as two-phase material is practically insensitive to the elastic heterogeneity of the isotropic phases. Fluctuations of phase eigenstrain fields - deviations from phase averages, have been elucidated to be the factor responsible for buildup of "stored" coherency energy - nonzero even after complete decomposition of austenitic phase. Experimental data for NiTi alloy show that the stored coherency energies $\phi_{\alpha}^{\text {st }}\left(\phi_{\alpha}^{\text {st }} \geq 0\right)$ can have even larger values than respective interaction energies $\phi_{\alpha \beta}^{(0) \text { coh }}$, as can be found upon comparison of difference in entries $\phi_{2}^{\text {st }}-\phi_{3}^{\text {st }}=1510 \mathrm{~J} / \mathrm{kg}\left(\Delta u_{3}^{0}-\Delta u_{2}^{0}\right)$ with entry for $\phi_{12}=1062 \mathrm{~J} / \mathrm{kg} \quad\left(\phi_{12}=\phi_{12}^{(0) \mathrm{coh}}\right)$, cf. Table 5.5 in Ziolkowski [32]. Thus in general it cannot be neglected as it considerably influences, e.g., characteristic phase transition temperatures of SMA material. This information can prove to be of practical value for manufacturers of SMA materials, who by formation of adequate field of fluctuations of phase eigenstrains during thermomechanical processing of SMA material can obtain required, useful functional properties. The micromechanical analysis presented in this work makes grounding step in development of unified pseudoelasticity and one-way memory model of SMA materials based on multiphase macroelement $(n>2)$.

The present study allows for drawing important conclusion that it is reasonable to adopt the Gibbs function of SMA material which is consistent with the following general form, cf. also (1), (73), (27), (75),

$$
\begin{aligned}
g\left(\mathrm{Y}_{h}^{T \sigma}, z_{\gamma}\right)= & u_{1}^{0}-T s_{1}^{0}-\left(1-z_{1}\right) \pi_{0}^{f}(T)+c_{p}\left[\Delta T-T \ln \left(T / T_{0}\right)\right]-\frac{\Delta T \boldsymbol{\sigma} \cdot \boldsymbol{\alpha}}{\rho}-\overbrace{\sum_{\alpha=2}^{n} z_{\alpha} g_{\alpha}^{\mathrm{ult}}\left(Y_{h}^{\boldsymbol{\sigma}}\right)}^{\sigma \cdot \varepsilon^{\mathrm{pt} / \rho}} \\
& -\overbrace{\left[\sum_{\alpha=1}^{n} z_{\alpha} g_{\alpha}^{(0) \mathrm{el}}(\boldsymbol{\sigma})-0.5 \sum_{\alpha, \beta=1, \alpha \neq \beta}^{n} z_{\alpha} z_{\beta} g_{\alpha \beta}^{\mathrm{el}}\left(Y_{h}^{\sigma}, z_{\gamma}\right)\right]}^{+\phi^{\mathrm{coh}}=0.5 \sigma \cdot M \sigma / \rho} \\
& +\overbrace{\sum_{\alpha=2}^{n} z_{\alpha} \phi_{\alpha}^{\mathrm{coh}}\left(h, z_{\gamma}\right)+0.5 \sum_{\alpha, \beta=1, \alpha \neq \beta}^{n} z_{\alpha} z_{\beta} \phi_{\alpha \beta}^{\mathrm{coh}}\left(h, z_{\gamma}\right)} .
\end{aligned}
$$

The mnemonic symbols $\mathrm{Y}_{\mathrm{h}}^{\mathrm{T} \sigma}$, $\mathrm{Y}_{\mathrm{h}}^{\sigma}$ denote specific subsets of independent state parameters external and internal ( $h$ hidden) in addition to $z_{\alpha}$. The internal variables describe the microstructure geometry of SMA macroelement. The $g_{\alpha}^{\text {ult }}(\alpha=2, \ldots, n)$ represents the work of macroscopic stress $\sigma$ exerted upon the ultimate phase strain $\kappa_{\alpha}$,

$$
\begin{aligned}
& g_{\alpha}^{\text {ult }}\left(\mathrm{Y}_{\mathrm{h}}^{\sigma}\right) \equiv \boldsymbol{\sigma} \cdot \boldsymbol{\kappa}_{\alpha} / \rho, \quad \rho \phi_{\alpha \beta}^{\mathrm{coh}} \equiv \rho \phi_{\alpha \beta}^{(0) \mathrm{coh}}-\left[\left(\boldsymbol{\Gamma}_{\alpha}-\boldsymbol{\Gamma}_{\beta}\right) \cdot \boldsymbol{\sigma}_{\alpha}^{(\mathrm{f}-\mathrm{in})}+\left(\boldsymbol{\Gamma}_{\beta}-\boldsymbol{\Gamma}_{\alpha}\right) \cdot \boldsymbol{\sigma}_{\beta}^{(\mathrm{f}-\mathrm{in})}\right] \\
& \{T, \boldsymbol{\sigma}, h\} \equiv \mathrm{Y}_{\mathrm{h}}^{\mathrm{T} \sigma}, \quad\{\boldsymbol{\sigma}, h\} \equiv \mathrm{Y}_{\mathrm{h}}^{\sigma} .
\end{aligned}
$$

The coefficients of macroscopic elastic energy $g_{\alpha \beta}^{(0) e l}\left(\mathrm{Y}_{h}^{\sigma}, z_{\gamma}\right)$ depend on $h$ through the dependence of the SEIM on $h$. The coherence coefficients $\phi_{\alpha \beta}^{\text {coh }}$ are additionally altered by $h$ due to the dependence of $\boldsymbol{\Gamma}_{\alpha}$ and $\boldsymbol{\sigma}_{\alpha}^{(\mathrm{f}-\mathrm{in})}$ on $h$.

Important aspect of the studies on reaching reasonable estimates of coherency energy originates from the concept that phase transitions are initiated at unstable equilibrium states. The direct consequence of this presumption is that specific, adopted form of thermodynamic potential immediately dictates the specific form of phase transition criteria. The issues of thermodynamic instability of phases in $n$-phases SMA macroelement and resulting from this phase transition criteria will be presented in a separate work.

Acknowledgements Support of the Polish Ministry of Science and Higher Education under Project No. N 501224537 is sincerely acknowledged. 
Open Access This article is distributed under the terms of the Creative Commons Attribution 4.0 International License (http:// creativecommons.org/licenses/by/4.0/), which permits unrestricted use, distribution, and reproduction in any medium, provided you give appropriate credit to the original author(s) and the source, provide a link to the Creative Commons license, and indicate if changes were made.

\section{References}

1. Abeyaratne, R., Knowles, J.K.: On the driving traction acting on a surface of strain discontinuity in a continuum. J. Mech. Phys. Solids 38, 345-360 (1990)

2. Bernardini, D.: On the macroscopic free energy functions for shape memory alloys. J. Mech. Phys. Solids 49, 813-837 (2001)

3. Bernardini, D., Pence, T.J.: Shape-memory materials, modeling. In: Schwartz, M.J. (ed.) Encyclopedia of Smart Materials, vol. 1, 2nd edn, pp. 964-980. Wiley, London (2002)

4. Bernardini, D., Masiani, R.: New micromechanical estimates of the interaction energy for shape memory alloys modeled by a two-phases microstructure. Solids Math, Mech (2014). doi:10.1177/1081286514562291

5. Dvorak, G.J., Benveniste, Y.: On transformation strains and uniform fields in multiphase elastic media. Proc. R. Soc. Lond. A 437, 291-310 (1992)

6. Dvorak, G.J.: Micromechanics of Composite Materials. Springer, New York (2013)

7. Follansbee, P.S.: Fundamentals of Strength-Principles, Experiment, and Application of an Internal State Variable Constitutive Model. The Minerals, Metals, \& Materials Society, Wiley, Hoboken (2014)

8. Hill, R.: Elastic properties of reinforced solids: some theoretical principles. J. Mech. Phys. Solids 11, 357-372 (1963)

9. Lagoudas, D.C., Entchev, P.B., Popov, P., Patoor, E., Brinson, L.C., Gao, X.: Shape memory alloys. Part II: modeling of polycrystals. Mech. Mater. 38, 430-462 (2006)

10. Lagoudas, D.C. (ed.): Shape Memory Alloys Modeling and Engineering Applications. Springer, New York (2008)

11. Levin, V.M.: On the coefficients of thermal expansion of heterogeneous materials. Mech. Solids 2, 58-61 (1967)

12. Milton, G.W.: Theory of Composites, Cambridge Monographs on Applied and Computational Mathematics. Cambridge University Press, Cambridge (2002)

13. Müller, I., Seelecke, S.: Thermodynamic aspects of shape memory alloys. Math. Comput. Model. 34, 1307-1355 (2001)

14. Nemat-Nasser, S., Hori, M.: Micromechanics: Overall Properties of Heterogeneous Materials. North-Holland, Amsterdam (1993)

15. Nishida, M., Kawano, H., Nishiura, T., Inamura, T.: Self-accommodation of B19/ martensite in Ti-Ni shape memory alloysPart I. Morphological and crystallographic studies of the variant selection rule. Philos. Mag. 92(17), 2215-2233 (2012)

16. Otsuka, K., Wayman, C.M. (eds.): Shape Memory Materials. Cambridge University Press, Cambridge (1998)

17. Patoor, E., Eberhardt, A., Berveiller, M.: Micromechanical modelling of superelasticity in shape memory alloys. J. Phys. IV C16, 277-292 (1996)

18. Peultier, B., Ben Zineb, T., Patoor, E.: Macroscopic constitutive law of shape memory alloy thermomechanical behaviour. Application to structure computation by FEM. Mech. Mater. 38, 510-524 (2006)

19. Popov, P., Lagoudas, D.C.: A 3-D constitutive model for shape memory alloys incorporating pseudoelasticity and detwinning of self-accommodated martensite. Int. J. Plast. 23, 1679-1720 (2007)

20. Raniecki, B., Lexcellent, C., Tanaka, K.: Thermodynamic models of pseudoelastic behaviour of shape memory alloys. Arch. Mech. 44(3), 261-284 (1992)

21. Raniecki, B., Tanaka, K.: On the thermodynamic driving force for coherent phase transformations. Int. J. Eng. Sci. 32(12), 1845-1858 (1994)

22. Raniecki, B., Lexcellent, C.: $R_{L}$-models of pseudoelasticity and their specification for some shape memory solids. Eur. J. Mech. A/Solids 13(1), 21-50 (1994)

23. Raniecki, B., Lexcellent, C.: Thermodynamics of isotropic pseudoelasticity in shape memory alloys. Eur. J. Mech. A/Solids 13(1), 185-205 (1998)

24. Raniecki, B., Tanaka, K., Ziółkowski, A.: Testing and modeling of NiTi SMA at complex stress state—selected results of Polish-Japanese Research Cooperation, Material Sciences Research Internal, Special Technical Publication, vol. 2, pp. 327-334 (2001)

25. Rychlewski, J.: Unconventional approach to linear elasticity. Arch. Mech. 47(2), 149-171 (1995)

26. Rosen, B.W., Hashin, Z.: Effective thermal expansion coefficients and specific heats of composite materials. Int. J. Eng. Sci. 8, 157-173 (1970)

27. Sun, Q.P., Hwang, K.C.: Micromechanics constitutive description of thermoelastic martensitic transformation. In: Huchinson, J., Wu, T.W. (eds.) Advances in Applied Mechanics, vol. 31, pp. 249-298. Academic Press, New York (1994)

28. Volokh, K.Y.: Stresses in growing soft tissues. Acta Biomater. 2, 493-504 (2006)

29. Walpole, L.J.: Elastic behaviour of composite materials: theoretical foundations. In: Yih, C.S. (ed.) Advances in Applied Mechanics, vol. 21, pp. 169-242. Academic Press, New York (1981)

30. Xiao Guo, Z. (ed.): Multiscale Materials Modeling Fundamentals and Applications. Woodhead Publishing Limited, Cambridge (2007)

31. Ziółkowski, A., Raniecki, B.: On the free energy function for Shape Memory Alloys treated as a two-phase continuum. Arch. Mech. 51(6), 785-911 (1999)

32. Ziółkowski, A.: Pseudoelasticity of Shape Memory Alloys, Theory and Experimental Studies. Butterworth-Heinemann, Amsterdam (2015) 